\title{
COLD DARK MATTER SUBSTRUCTURE AND GALACTIC DISKS. II. DYNAMICAL EFFECTS OF HIERARCHICAL SATELLITE ACCRETION
}

\author{
Stelios KaZantzidis ${ }^{1,2,3}$, Andrew R. Zentner ${ }^{4}$, Andrey V. Kravtsov ${ }^{5,6,7}$, JAmes S. Bullock ${ }^{8,9}$, AND \\ Victor P. DEBATTISTA ${ }^{10,11}$ \\ ${ }^{1}$ Center for Cosmology and Astro-Particle Physics, Ohio State University, Columbus, OH 43210, USA; stelios@mps.ohio-state.edu \\ ${ }^{2}$ Department of Physics, Ohio State University, Columbus, OH 43210, USA \\ ${ }^{3}$ Department of Astronomy, Ohio State University, Columbus, OH 43210, USA \\ ${ }^{4}$ Department of Physics and Astronomy, University of Pittsburgh, Pittsburgh, PA 15260 USA; Zentner@pitt.edu \\ ${ }^{5}$ Kavli Institute for Cosmological Physics, University of Chicago, Chicago, IL 60637, USA \\ ${ }^{6}$ Department of Astronomy \& Astrophysics, University of Chicago, IL 60637, USA \\ 7 The Enrico Fermi Institute, University of Chicago, Chicago, IL 60637, USA; andrey@ oddjob.uchicago.edu \\ ${ }^{8}$ Center for Cosmology, University of California at Irvine, Irvine, CA 92697, USA \\ ${ }^{9}$ Department of Physics \& Astronomy, University of California at Irvine, Irvine, CA 92697, USA; bullock@ uci.edu \\ ${ }^{10}$ Center for Astrophysics, University of Central Lancashire, Preston PR1 2HE, UK; vpdebattista@uclan.ac.uk \\ Received 2009 February 11; accepted 2009 June 4; published 2009 July 17
}

\begin{abstract}
We perform a set of fully self-consistent, dissipationless $N$-body simulations to elucidate the dynamical response of thin galactic disks to bombardment by cold dark matter (CDM) substructure. Our method combines (1) cosmological simulations of the formation of Milky Way (MW)-sized CDM halos to derive the properties of substructure, and (2) controlled numerical experiments of consecutive subhalo impacts onto an initially thin, fully formed MW-type disk galaxy. The present study is the first to account for the evolution of satellite populations over cosmic time in such an investigation of disk structure. In contrast to what can be inferred from statistics of the $z=0$ surviving substructure, we find that accretions of massive subhalos onto the central regions of host halos, where the galactic disks reside, since $z \sim 1$ should be common. One host halo accretion history is used to initialize the controlled simulations of satellite-disk encounters. The specific merger history involves six dark matter substructures, with initial masses in the range $\sim 20 \%-60 \%$ of the disk mass and of comparable size to the disk, crossing the central regions of their host in the past $\sim 8$ Gyr. We show that these accretion events severely perturb the thin galactic disk and produce a wealth of distinctive dynamical signatures on its structure and kinematics. These include (1) considerable thickening and heating at all radii, with the disk thickness and velocity ellipsoid nearly doubling at the solar radius; (2) prominent flaring associated with an increase in disk thickness greater than a factor of 4 in the disk outskirts; (3) surface density excesses at large radii, beyond $\sim 5$ disk scale lengths, resembling those of the observed antitruncated disks; (4) long-lived, lopsidedness at levels similar to those measured in observational samples of disk galaxies; and (5) substantial tilting. The interaction with the most massive subhalo in the simulated accretion history drives the disk response while subsequent bombardment is much less efficient at disturbing the disk. We also explore a variety of disk and satellite properties that influence these responses. We conclude that substructure-disk encounters of the kind expected in the $\Lambda \mathrm{CDM}$ paradigm play a significant role in setting the structure of disk galaxies and driving galaxy evolution.
\end{abstract}

Key words: cosmology: theory - dark matter - galaxies: evolution - galaxies: formation - galaxies: structure methods: numerical

Online-only material: color figures

\section{INTRODUCTION}

Hierarchical models of cosmological structure formation, such as the currently favored cold dark matter (CDM) paradigm (e.g., White \& Rees 1978; Blumenthal et al. 1984), generically predict substantial amounts of substructure in the form of small, dense, self-bound subhalos orbiting within the virialized regions of larger host halos (e.g., Ghigna et al. 1998, 2000; Tormen et al. 1998; Moore et al. 1999; Klypin et al. 1999b). Observational probes of substructure abundance thus constitute fundamental tests of the CDM model. Recently, a growing body of evidence has confirmed the hierarchical build-up of galaxysized dark matter halos with the discovery of tidal streams and complex stellar structures in the Milky Way (MW; e.g., Ibata et al. 1994, 2001b; Yanny et al. 2000; Newberg et al. 2002;

\footnotetext{
${ }^{11}$ RCUK Fellow.
}

Majewski et al. 2003; Martin et al. 2004; Martínez-Delgado et al. 2005; Grillmair \& Dionatos 2006; Belokurov et al. 2006), the Andromeda galaxy (M31; Ibata et al. 2001a, 2007; Ferguson et al. 2002, 2005; Kalirai et al. 2006), and beyond the Local Group (e.g., Malin \& Hadley 1997; Shang et al. 1998; Peng et al. 2002; Forbes et al. 2003; Pohlen et al. 2004).

A significant fraction of observed galaxies have diskdominant morphology with roughly $70 \%$ of Galaxy-sized dark matter halos hosting late-type systems (e.g., Weinmann et al. 2006; Choi et al. 2007). Owing to the lack of a significant luminous component associated with most subhalos in Galaxy-sized host halos (e.g., Klypin et al. 1999b; Moore et al. 1999), information regarding the amount of substructure in these systems may be obtained via its gravitational influence on galaxy disks. Despite the small contribution of substructure to the total mass of the host (e.g., Ghigna et al. 2000), a considerable number of subhalos are expected within the virialized region of a Galaxy- 
sized CDM halo at any given epoch. If a large population of satellites exists, it may tidally disturb the host galactic disk, possibly leading to the imprint of distinctive dynamical signatures on its structure and kinematics.

Theoretical studies set within the CDM paradigm have convincingly shown that the accretion of massive substructures is commonplace during the formation of Galaxy-sized halos (e.g., Lacey \& Cole 1993; Zentner \& Bullock 2003; Purcell et al. 2007; Stewart et al. 2008) and that typical subhalo orbits are highly eccentric (e.g., Ghigna et al. 1998; Tormen et al. 1998; Zentner et al. 2005a; Benson 2005). These facts suggest that passages of massive satellites near the center of the host potential, where the galactic disks reside, should be common in CDM models. Such accretion events are expected to perturb the fragile circular orbits of disk stars by depositing large amounts of orbital energy into random stellar motions, gradually heating the disk and increasing its scale height.

Yet, many disk galaxies are observed to be cold and thin, with average axial ratios of radial scale lengths to vertical scale heights, $R_{d} / z_{d}$, in the range $\sim 4-5$ (e.g., de Grijs 1998; Bizyaev \& Mitronova 2002; Kregel et al. 2002; Yoachim \& Dalcanton 2006). In addition, recent studies of edge-on disk galaxies using the Sloan Digital Sky Survey (SDSS) database have revealed a notable fraction of "super-thin" bulgeless disks with much larger axial ratios (Kautsch et al. 2006).

In the case of the MW, measurements of the disk scale height at the solar radius obtained using a variety of methods, including star counts and mass modeling, indicate that the Galaxy comprises a thin, stellar disk with an exponential scale height of $h_{z} \simeq 300 \pm 50$ pc (e.g., Kent et al. 1991; Dehnen \& Binney 1998; Mendez \& Guzman 1998; Larsen \& Humphreys 2003; Widrow \& Dubinski 2005; Jurić et al. 2008). Furthermore, the age-velocity dispersion relation of disk stars in the solar neighborhood suggests that a significant fraction of the thin disk of the MW was in place by $z \sim 1$ (e.g., Wyse 2001; Quillen \& Garnett 2000; Nordström et al. 2004; Seabroke \& Gilmore 2007). The existence of such an old, thin stellar disk, as established by the age distribution of disk stars, may imply an absence of satellite accretion events over the past $\sim 8$ Gyr. Such an extended period of quiescent dynamical evolution is difficult to reconcile with the hierarchical assembly of structure prescribed by the $\Lambda \mathrm{CDM}$ cosmological model. Although hydrodynamical simulations of disk galaxy formation have offered some insights into accommodating observational facts that challenge the CDM paradigm (e.g., Abadi et al. 2003), the detailed dynamical response of galactic disks to halo substructure in a cosmological context remains poorly understood.

Significant theoretical effort, including both semianalytic modeling (Toth \& Ostriker 1992; Benson et al. 2004; Hopkins et al. 2008) and numerical simulations (Quinn \& Goodman 1986; Quinn et al. 1993; Walker et al. 1996; Huang \& Carlberg 1997; Sellwood et al. 1998; Velazquez \& White 1999; Font et al. 2001; Ardi et al. 2003; Gauthier et al. 2006; Hayashi \& Chiba 2006; Read et al. 2008; Villalobos \& Helmi 2008; Purcell et al. 2009) has been devoted to quantifying the resilience of galactic disks to infalling satellites. Although valuable in several respects, these earlier investigations could not capture fully the amount of global dynamical evolution induced in thin-disk galaxies by substructure in the context of the $\Lambda \mathrm{CDM}$ model. While a detailed comparison to previous work is presented in Section 6, we mention at the outset that each of the aforementioned numerical studies suffered from at least one critical shortcoming by either: (1) not being fully self-consistent, modeling various components of the primary disk galaxy and/or the satellites as rigid potentials, a choice which leads to overestimating the damage caused to the disk; (2) focusing on experiments with infalling systems on nearly circular orbits that are poor approximations of the highly eccentric orbits typical of CDM substructure; (3) adopting galactic disks that are much thicker compared to typical thin disks including the old, thin stellar disk of the MW; (4) modeling the compact, baryonic cores of accreting systems exclusively and neglecting the more diffuse and extended dark matter component; and (5) considering the encounters of individual satellites with galactic disks, despite the CDM expectations of numerous accretion events over the history of a galaxy.

Regarding the final point, notable exceptions were the studies by Font et al. (2001) and Gauthier et al. (2006) which examined the dynamical evolution of a stellar disk in the presence of a large ensemble of dark matter subhalos. Both contributions reported negligible tidal effects on the global structure of the disks. However, these investigations had the drawback of adopting the $z=0$ surviving substructure present in a Galaxy-sized CDM halo, and thus not accounting for past encounters of systems with the galactic disk during the evolution of the satellite populations. This point is critical because as subhalos on highly eccentric orbits continuously lose mass, the number of massive satellites with small orbital pericenters that are most capable of severely perturbing the disk declines with redshift so that few would be present at $z=0$ (e.g., Zentner \& Bullock 2003; Kravtsov et al. 2004; Gao et al. 2004). Establishing the role of halo substructure in shaping the fine structure of disk galaxies clearly requires a more realistic treatment of its evolution over cosmic time.

Our aim is to improve upon these shortcomings and extend consideration to the rich structure of perturbed galactic disks. This paper is the second in a series elucidating the effects of halo substructure on thin-disk galaxies in the context of the prevailing CDM paradigm. Kazantzidis et al. (2008, hereafter Paper I) focused on the generic morphological signatures induced in the disk by a typical $\Lambda \mathrm{CDM}$-motivated satellite accretion history, while the present paper discusses the dynamical response of the galactic disk subject to bombardment by the same population of dark matter subhalos. We implement a two-step strategy in an effort to overcome some of the drawbacks of past studies. First, we analyze dissipationless, cosmological simulations of the formation of Galaxy-sized CDM halos to derive the accretion histories and properties of substructure populations. This information is subsequently used to seed collisionless, controlled numerical experiments of consecutive satellite impacts onto $\mathrm{N}$-body realizations of fully formed, thin-disk galaxies. Given the outstanding issues regarding disk galaxy formation in CDM cosmogonies (e.g., Mayer et al. 2008) as well as the inadequacies of the current generation of cosmological simulations to resolve all dynamical scales and physical processes relevant to satellite-disk interactions, this strategy is most appropriate. As in Paper I, we model the infalling systems as pure dark matter subhalos and focus exclusively on the evolution of the stellar material in the disk itself.

Our contribution improves upon earlier studies in several important respects. First and foremost, we examine the response of galactic disks to subhalo populations that are truly representative of those accreted and possibly destroyed in the past. As such, we mitigate the biases in the incidence of accretion events 
and properties of infalling satellites induced by considering only the $z=0$ substructure of a CDM halo. Specifically, we extract merger histories of host halos since $z \sim 1$ and study the ramifications of such accretion events for disk structure. As we illustrate below, our methodology results in a substantially larger number of potentially damaging subhalo-disk encounters than previously considered. Second, we construct self-consistent satellite models whose properties are culled directly from the same cosmological simulations of Galaxy-sized CDM halos. This obviates the need for arbitrary assumptions regarding the numbers, masses, internal structures, orbital parameters, and accretion times of the infalling subhalos.

Lastly, we employ multicomponent disk galaxy models that are derived from explicit distribution functions, are motivated by the $\Lambda C D M$ paradigm, and are flexible enough to permit detailed modeling of actual galaxies such as the MW and M31 by incorporating a wide range of observational constraints. These properties in synergy with the adopted high mass and force resolution enable us to construct realistic, equilibrium $N$-body models of thin-disk galaxies and study their dynamical response to encounters with satellites. For our primary simulation set, we use a best-fit model for the present-day structure of the MW and employ an initial disk scale height of $z_{d}=400 \mathrm{pc}$ which is consistent with that of the old, thin stellar disk of the Galaxy. Although we utilize a model for the MW, we do emphasize that our simulation campaign is neither designed to follow the evolution of nor to draw specific conclusions about the Galaxy or any other particular system. Given the complex interplay of effects (e.g., gas cooling, star formation, chemical evolution) relevant to the formation and evolution of spiral galaxies, our collisionless simulations aim for generic features of the evolution of a thin galactic disk subject to bombardment by CDM substructure.

Our work establishes that the types of merger histories expected in $\Lambda \mathrm{CDM}$ can substantially perturb the structure of a cold, stellar disk. We demonstrate that cosmological halo assembly via multiple accretion events is capable of generating a wealth of distinctive dynamical signatures in the structural and kinematic properties of disk stars. These include pronounced thickening and heating, prominent flaring and tilting, surface density excesses that develop in the outskirts similar to those of observed antitruncated disks, and lopsidedness at levels similar to those measured in the observed galaxies. Our findings suggest that details of the galaxy assembly process may be imprinted on the dynamics of stellar populations and corroborate the concept of accretion-induced galaxy evolution. We also show that the global dynamical response of a galactic disk to interactions with satellites depends sensitively on a variety of parameters including the initial disk thickness, the presence of a bulge component in the primary disk, the internal density distribution of the infalling systems, and the relative orientation of disk and satellite angular momenta.

The remainder of this paper is organized as follows. In Section 2, we describe the methods employed in this study. Section 3 contains the results regarding the dynamical signatures induced in a thin galactic disk by a typical $\Lambda C D M$ accretion history. In Section 4, we study various factors that could influence the response of disk galaxies to satellite accretion events. Implications and extensions of our findings along with a comparison to previous work are presented in Sections 5 and 6. Finally, in Section 7, we summarize our main results and conclusions. Throughout this work we use the terms "satellites," "subhalos," and "substructures" interchangeably to indicate the distinct, gravitationally bound objects that we use as the basis for the controlled satellite-disk encounter simulations.

\section{METHODS}

A thorough description of the adopted methodology is given in Paper I. For completeness, we provide a summary of our approach here and refer the reader to Paper I for details.

\subsection{Hierarchical Cosmological Simulations}

We analyze high-resolution, collisionless cosmological simulations of the formation of four Galaxy-sized halos in a flat $\Lambda$ CDM cosmological model with parameters $\left(\Omega_{m}, \Omega_{\Lambda}, \Omega_{b}, h, \sigma_{8}\right)=(0.3,0.7,0.043,0.7,0.9)$. The simulations were performed with the Adaptive Refinement Tree (ART) $N$-body (Kravtsov et al. 1997; Kravtsov 1999). The host halos that we considered come from two different simulations. Halos " $\mathrm{G}_{1}$," " $\mathrm{G}_{2}$," and " $\mathrm{G}_{3}$ " all formed within a cubic box of length $25 h^{-1} \mathrm{Mpc}$ on a side, while halo " $\mathrm{G}_{4}$ " formed in a cubic volume of $20 h^{-1} \mathrm{Mpc}$ on a side. The mass and force resolution of these simulations as well as various properties of halos $G_{1}-G_{4}$ and their substructures were discussed in Paper I and in previous literature (Klypin et al. 2001; Kravtsov et al. 2004; Zentner et al. 2005b; Prada et al. 2006; Gnedin \& Kravtsov 2006). We identify halos (outside hosts $\mathrm{G}_{1}-\mathrm{G}_{4}$ ) and substructures using a variant of the Bound Density Maxima algorithm (Klypin et al. 1999a) and we have constructed detailed accretion histories for each host halo and orbital tracks for all subhalos. The specifics of this analysis can be found in Paper I and are based largely on Kravtsov et al. (2004).

Our conventions are as follows. We adopt a mean overdensity of 337 to define the virial radius, $r_{\text {vir }}$, and the corresponding virial mass, $M_{\mathrm{vir}}$, for each host halo at $z=0$. Halos $\mathrm{G}_{1}$ through $\mathrm{G}_{4}$ have $r_{\mathrm{vir}} \simeq(234,215,216,230) h^{-1} \mathrm{kpc}$ and $M_{\text {vir }} \simeq(1.5,1.1,1.1,1.4) \times 10^{12} h^{-1} M_{\odot}$, respectively. All four of these halos accrete only a small fraction of their final mass and experience no major mergers at $z \lesssim 1$, and are thus likely to host a disk galaxy. Moreover, their accretion histories are typical of systems in this mass range (e.g., Wechsler et al. 2002). We have chosen $G_{1}$ as our fiducial case for the satellitedisk interaction experiments described in Section 2.4.

\subsection{Interactions Between Substructures and Disks in CDM}

We investigate the dynamical response of thin galactic disks to interactions with CDM substructure incorporating for the first time a model that accounts for its evolution over cosmic time. Previous related studies (e.g., Font et al. 2001; Gauthier et al. 2006) only utilized subhalo populations at $z=0$, rather than a complete merger history, to seed simulations of satellitedisk encounters in a cosmological context. This procedure has the drawback of eliminating from consideration those massive satellites that, prior to $z=0$, cross through the central regions of their hosts, where the galactic disks reside. Such systems can potentially produce strong tidal effects on the disk, but are unlikely to constitute effective perturbers at $z=0$ as they suffer substantial mass loss (or even become disrupted) during their orbital evolution precisely because of their forays into the central halo (e.g., Zentner \& Bullock 2003; Kravtsov et al. 2004; Gao et al. 2004; Zentner et al. 2005a; Benson 2005). Accounting for the impact of these relatively short-lived objects on the global dynamical response of galactic disks is the major improvement we introduce in the present study. 


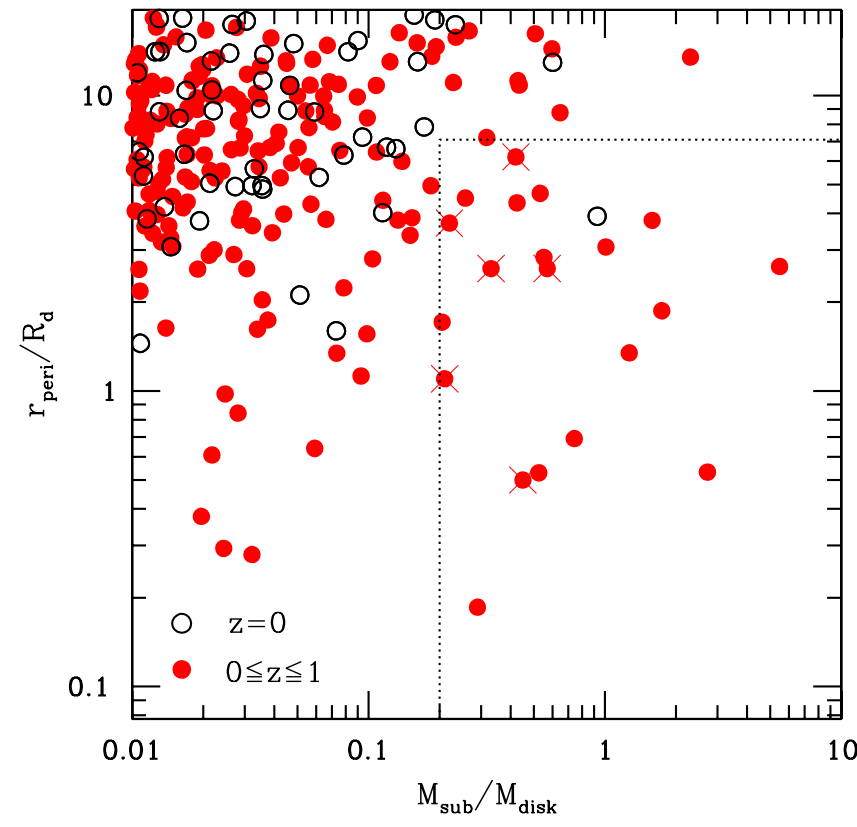

Figure 1. Scatter plot of mass vs. pericentric distance for subhalos identified in four Galaxy-sized dark matter halos formed in the $\Lambda$ CDM cosmology. Results are shown after scaling the virial quantities of all host halos to the corresponding values of the primary disk galaxy model used in the controlled satellite-disk encounter simulations of Section 2.4. Subhalo masses and pericenters are assigned according to the description in the text and are presented in units of the mass, $M_{\text {disk }}=3.53 \times 10^{10} M_{\odot}$, and scale length, $R_{d}=2.82 \mathrm{kpc}$, respectively, of the disk in the same galaxy model. The filled symbols refer to systems that cross within a (scaled) infall radius of $r_{\text {inf }}=50 \mathrm{kpc}$ from their host halo center since a redshift $z=1$. Symbols with crosses correspond to the specific subhalos in host halo $\mathrm{G}_{1}$ used to seed the controlled simulations of satellite-disk interactions. Open symbols refer to the $z=0$ surviving substructures. The dotted lines mark the so-called "danger zone" with $M_{\text {sub }} \gtrsim 0.2 M_{\text {disk }}$ and $r_{\text {peri }} \lesssim 20 \mathrm{kpc}$ corresponding to infalling subhalos that are capable of substantially perturbing the disk. Close encounters between massive substructures and galactic disks since $z=1$ should be common occurrences in $\Lambda$ CDM. In contrast, very few satellites in present-day subhalo populations are likely to have a significant dynamical impact on the disk structure.

(A color version of this figure is available in the online journal.)

Figure 1 serves as a dramatic illustration of this element of our modeling. This figure is a scatter plot of mass versus pericentric distance for two different substructure populations within host halos $\mathrm{G}_{1}-\mathrm{G}_{4}$. The masses and pericenters of all subhalos have been scaled to the mass, $M_{\text {disk }}=3.53 \times 10^{10} M_{\odot}$, and radial scale length, $R_{d}=2.82 \mathrm{kpc}$, of the disk in the primary galaxy model used in the controlled satellite-disk encounter simulations of Section 2.4. For the purposes of this presentation, we have also scaled the virial quantities $\left(M_{\mathrm{vir}}\right.$ and $\left.r_{\mathrm{vir}}\right)$ of all four Galaxy-sized host halos to the total mass, $M_{h}=7.35 \times 10^{11} M_{\odot}$, and tidal radius, $R_{h}=244.5 \mathrm{kpc}$, of the parent dark matter halo in the same galaxy model. The full list of parameters pertaining to the primary disk galaxy will be listed in Section 2.3.3.

The first substructure population of Figure 1 comprises systems that cross within a (scaled) infall radius of $r_{\text {inf }}=50 \mathrm{kpc}$ from their host halo center since a redshift $z=1$. This selection is fixed empirically to identify orbiting satellites that approach the central regions of the host potential and are thus likely to have a significant dynamical impact on the disk structure (Paper I). We assign masses to the satellites of this group at the simulation output time nearest to the first inward crossing of $r_{\text {inf }}$. As we discuss below, we define this to be the epoch that our controlled simulations initiate. The corresponding pericenters are computed from the orbit of a test particle in a static Navarro et al. (1996, hereafter NFW) potential whose properties match those of the host CDM halo at the time of $r_{\text {inf }}$. We note that a single distinct object of this population may be recorded multiple times as one subhalo may undergo several passes through the central regions of its host with different masses and pericenters. Many of these satellites suffer substantial mass loss or even become tidally disrupted prior to $z=0$.

The second subhalo population consists of the $z=0$ surviving substructures. Their pericenters are also estimates based on the orbit of a test particle in a static NFW potential whose properties match those of the host CDM halo at $z=0$. The dotted line in Figure 1 encloses an area in the $M_{\text {sub }}-r_{\text {peri }}$ plane corresponding to satellites more massive than $0.2 M_{\text {disk }}$ with pericenters of $r_{\text {peri }} \lesssim 20 \mathrm{kpc}\left(r_{\text {peri }} \lesssim 7 R_{d}\right)$. We refer to this area as the "danger zone." Subhalos within this area should be effective perturbers, but we intend this as a rough criterion to aid in illustrating our point.

Figure 1 demonstrates that the $z=0$ substructure populations contain very few massive systems on potentially damaging orbits. In fact, in all four of our host halos only one satellite can be identified inside the danger zone at $z=0$. As a consequence, the present-day substructure in a CDM halo likely plays only a minor role in driving the dynamical evolution of galactic disks. This conclusion is supported by Font et al. (2001) and Gauthier et al. (2006) who did consider the $z=0$ subhalo populations and reported negligible effects on the global disk structure.

On the other hand, the danger zone contains numerous satellites that have penetrated deeply into their host halo since $z=1$ : on average, $\sim 5$ systems more massive than $0.2 M_{\text {disk }}$ pass close to the center of their hosts with $r_{\text {peri }} \lesssim 20 \mathrm{kpc}$ in the past $\sim 8$ Gyr. We also stress that three of the host halos have accreted at least one satellite more massive than the disk itself since $z=1$. This finding is corroborated by analysis of simulations with much better statistics (Stewart et al. 2008). Overall, the results in Figure 1 indicate that close encounters between massive subhalos and galactic disks since $z=1$ should be common occurrences in the $\Lambda \mathrm{CDM}$ cosmological model. It is thus important to quantify and account for such interactions when the goal is to investigate the cumulative dynamical effects of halo substructure on galactic disks.

\subsection{Initial Conditions for Satellite-Disk Encounters}

Initializing the controlled experiments of subhalo-disk encounters involves: (1) identifying relevant substructures in the cosmological simulations and recording their properties (mass functions, internal structures, orbital parameters, and accretion times); and (2) constructing $N$-body realizations of disk galaxy and satellite models. In what follows, we describe each of these steps.

\subsubsection{Subhalo Selection Criteria}

We impose two criteria for selecting cosmological satellites for re-simulation (see also Paper I). We limit our search to systems that approach the central regions of halo $\mathrm{G}_{1}$ with (scaled) pericenters of $r_{\text {peri }} \lesssim 20 \mathrm{kpc}$ since $z=1$. This choice is motivated by the fact that subhalos with small orbital pericenters are expected to substantially perturb the galactic disk. Likewise, we select only subhalos that are a significant fraction of the disk mass, with (scaled) masses of $M_{\text {sub }} \gtrsim 0.2 M_{\text {disk }}$, as these will have the largest impact on the disk structure. We initiate our controlled re-simulations at the epoch when each selected subhalo first crossed a (scaled) infall radius of $r_{\text {inf }}=50 \mathrm{kpc}$ from the host halo center. 
Table 1

Parameters of the Satellite Models

\begin{tabular}{lccccccrrrrr}
\hline \hline Model & $z_{\text {acc }}$ & $M_{\text {sub }} / M_{\text {disk }}$ & $r_{\text {tid }} / R_{d}$ & $r_{\text {peri }} / R_{d}$ & $r_{\text {apo }} / r_{\text {peri }}$ & \multicolumn{1}{c}{$\begin{array}{c}\theta \\
\left({ }^{\circ}\right)\end{array}$} & $\begin{array}{r}V_{\text {sub }} \\
\left(\mathrm{km} \mathrm{s}^{-1}\right)\end{array}$ & $\epsilon_{J}$ & $(\alpha, \beta, \gamma)$ & $\begin{array}{c}r_{s} \\
(\mathrm{kpc})\end{array}$ \\
$(1)$ & $(2)$ & $(3)$ & $(4)$ & $(5)$ & \multicolumn{1}{c}{$(6)$} & $(7)$ & \multicolumn{1}{c}{$(8)$} & $(9)$ & $(10)$ \\
\hline S1 & 0.96 & 0.33 & 8.8 & 2.6 & 6.8 & 93.3 & 72.9 & 0.41 & $(0.12,3.85,1)$ & 2.1 & 11.6 \\
S2 & 0.89 & 0.57 & 7.6 & 2.6 & 6.0 & 86.6 & 70.9 & 0.46 & $(0.21,4.02,1)$ & 2.6 & 8.1 \\
S3 & 0.54 & 0.42 & 8.2 & 6.2 & 3.2 & 45.1 & 158.2 & 0.72 & $(0.38,3.72,1)$ & 2.2 & 10.6 \\
S4 & 0.32 & 0.45 & 7.0 & 0.5 & 20.6 & 117.7 & 19.6 & 0.16 & $(0.25,4.18,1)$ & 1.6 & 12.5 \\
S5 & 0.20 & 0.22 & 9.7 & 3.7 & 9.3 & 59.9 & 171.3 & 0.35 & $(0.16,3.94,1)$ & 1.8 & 15.2 \\
S6 & 0.11 & 0.21 & 8.2 & 1.1 & 19.6 & 144.5 & 89.6 & 0.17 & $(0.29,4.09,1)$ & 1.3 & 18.3 \\
\hline
\end{tabular}

Notes. Columns 2-9 record satellite properties at the epoch closest to when each subhalo first crossed within a (scaled) infall radius of $r_{\mathrm{inf}}=50 \mathrm{kpc}$ from the center of host halo $\mathrm{G}_{1}$. Columns 10-12 list structural parameters computed from the $N$-body realizations of satellite models used in the controlled subhalo-disk encounter simulations of Section 2.4. All entries are listed after scaling the virial quantities of halo $\mathrm{G}_{1}$ to the corresponding values of the parent halo in the fiducial disk galaxy model used in the controlled experiments. Note that values of orbital circularities and apocenter-to-pericenter ratios may deviate from those measured directly in the controlled simulations because the former are estimated from the satellite orbits in the potential of host halo $\mathrm{G}_{1}$. Column 1 : satellite model. Column 2: redshift at which the properties of cosmological satellites were recorded. Column 3: bound satellite mass in units of the mass of the disk, $M_{\text {disk }}=3.53 \times 10^{10} M_{\odot}$, in the fiducial galaxy model used in the controlled encounter simulations. Column 4: satellite tidal radius in units of the radial scale length of the fiducial disk, $R_{d}=2.82 \mathrm{kpc}$. Column 5: pericenter of the satellite orbit in units of $R_{d}$. Column 6: satellite orbital apocenter-to-pericenter ratio. Column 7: angle between the initial angular momenta of the satellite and the disk in degrees. This angle is defined so that $0^{\circ}<\theta<90^{\circ}$ corresponds to a prograde orbit and $90^{\circ}<\theta<180^{\circ}$ corresponds to a retrograde orbit. The cases where $\theta=0^{\circ}, \theta=90^{\circ}$, and $\theta=180^{\circ}$ represent a coplanar prograde, a polar, and a coplanar retrograde orbit, respectively. Column 8: satellite three-dimensional orbital velocity in $\mathrm{km} \mathrm{s}^{-1}$. Column 9: circularity of the orbit. This parameter is defined as $\epsilon_{J} \equiv J / J_{\text {circ }}(E)$, where $J$ is the satellite angular momentum and $J_{\text {circ }}(E)$ is the corresponding angular momentum for a circular orbit of the same energy $E$. Column 10: intermediate, outer, and inner slopes of the satellite density profile. Inner and outer slopes correspond to the asymptotic values. Column 11: scale radius of the satellite density profile in kpc. This radius is defined as the distance where the logarithmic slope is the average of the inner and outer slopes, $d \ln \rho(r) / d \ln r=-(\gamma+\beta) / 2$. Column 12: satellite concentration defined as $c \equiv r_{\text {tid }} / r_{\mathrm{s}}$.

Figure 1 shows that, on average, $\sim 5$ systems meet these two criteria for each host halo. Although at least one subhalo more massive than the disk in our controlled simulations is expected to cross the central regions of their hosts since $z \sim 1$ (Figure 1; Stewart et al. 2008), we explicitly ignore these interactions with $M_{\text {sub }} \gtrsim M_{\text {disk}}$. Our goal is to investigate the tidal effects of substructure on a well-preserved disk galaxy while such encounters may destroy the disk upon impact (Purcell et al. 2009).

These criteria result in a merger history of six accretion events, which we denote as S1-S6, for subsequent re-simulation over an $\approx 8 \mathrm{Gyr}$ period. These substructures correspond to the filled symbols with crosses seen in Figure 1 and their properties are summarized in Table 1. A critical reader may note that the internal properties and orbital parameters of subhalos in collisionless cosmological simulations, as those of the present study, may differ substantially from those in simulations with gasdynamics. Indeed, in hydrodynamical simulations of disk galaxy formation, the average satellite crossing the central regions of its host experiences additional mass loss due to disk shocking and both circularization of its orbit and decrease of its orbital inclination via dynamical friction against the disk (e.g., Quinn \& Goodman 1986; Peñarrubia et al. 2002; Meza et al. 2005). These criticisms are mitigated for S1-S6 as we identified these objects and recorded their properties well prior to their first crossing of the halo center.

Table 1 contains the main orbital and structural properties of cosmological satellites S1-S6. These properties have been discussed extensively in Paper I. Nonetheless, a few parameters are worthy of explicit note. First, the masses of these six substructures correspond to the upper limit of the mass function of observed satellites in the Local Group. For reference, the mass of the Large Magellanic Cloud (e.g., Schommer et al.
1992) is similar to that of our most massive subhalo S2. Moreover, satellites S1-S6 are spatially extended with their tidal radii, $r_{\text {tid }}$, encompassing a significant fraction of the disk itself $\left(r_{\text {tid }} \gtrsim 7 R_{d}\right)$. The entire disk will be subject to potential fluctuations as a result. Thus, the energy imparted by typical cosmological substructures may not be deposited locally at the point of impact, as assumed by Toth \& Ostriker (1992), but globally across the entire disk. Lastly, we note that most subhalos S1-S6 are on highly eccentric orbits and that the simulated accretion history includes nearly polar (S1, S2), prograde (S3, S5), and retrograde (S4, S6) encounters.

\subsubsection{Constructing N-body Realizations of Satellites}

Our controlled experiments employ satellite models which are constructed to match the internal structure of cosmological subhalos S1-S6. The density profiles of these systems are extracted at the simulation output closest to each subhalo's first inward crossing of $r_{\text {inf. }}$. We model all cosmological satellites with the general density profile law (Zhao 1996; Kravtsov et al. 1998)

$$
\rho(r)=\frac{\rho_{\mathrm{s}}}{\left(r / r_{\mathrm{s}}\right)^{\gamma}\left[1+\left(r / r_{\mathrm{s}}\right)^{\alpha}\right]^{(\beta-\gamma) / \alpha}} \quad\left(r \leqslant r_{\mathrm{tid}}\right),
$$

where $\rho_{s}$ sets the normalization of the density profile, $r_{\text {tid }}$ is the limiting radius of the satellite imposed by the host halo tidal field. The exponents $\gamma$ and $\beta$ denote the asymptotic inner and outer slopes of the profile, respectively. Exponent $\alpha$ parameterizes the transition between the inner and outer profiles. Lastly, at the scale radius $r_{s}$, the logarithmic slope is the average of the inner and outer slopes, $d \ln \rho(r) / d \ln r=-(\gamma+\beta) / 2$.

In practice, all subhalos are described well by Equation (1) with $\gamma=1$, so we fix this value and fit their structure by 


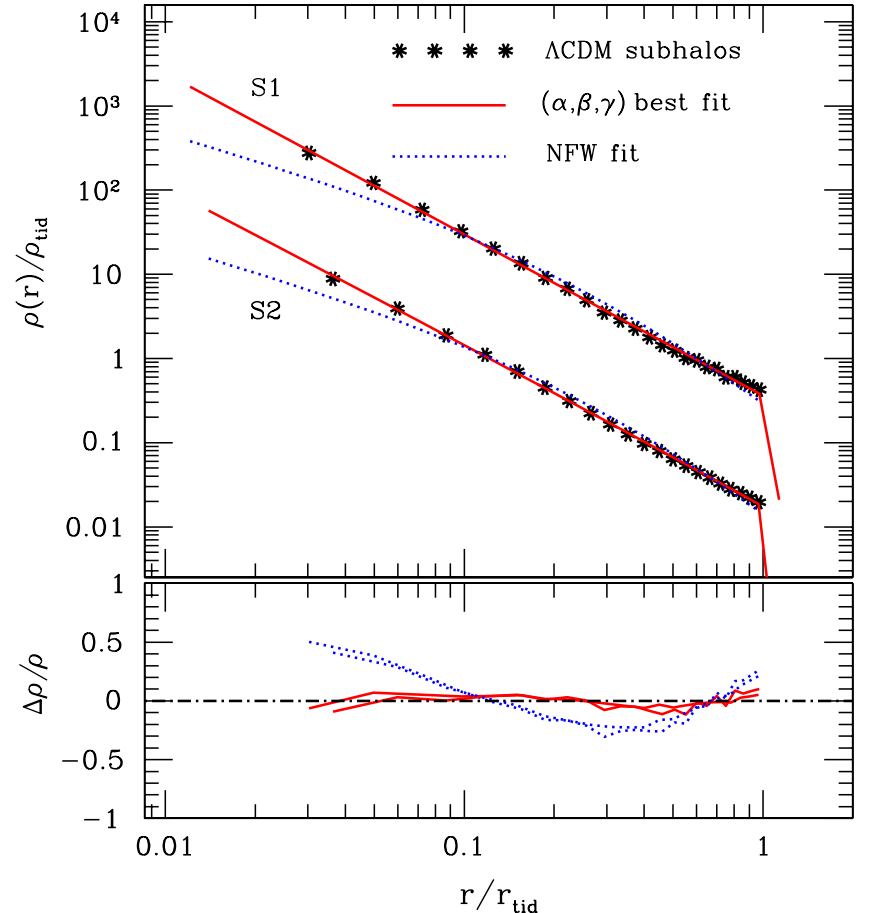

Figure 2. Upper panel: spherically averaged density profiles, $\rho(r)$, for representative cosmological satellites $\mathrm{S} 1$ and $\mathrm{S} 2$ as a function of radius in units of the tidal radius of each system, $r_{\text {tid }}$. Stars correspond to the subhalo profiles extracted directly from the cosmological simulation of host halo $\mathrm{G}_{1}$. The solid lines present fits to the density structure using a multiparameter $(\alpha, \beta, \gamma)$ density law, while the dotted lines show the corresponding NFW fits. The solid lines include an exponential cutoff at $r>r_{\text {tid }}$. All curves are plotted from the adopted force resolution $\left(2 \epsilon_{\mathrm{sub}}=300 \mathrm{pc}\right)$ outward and densities are normalized to the mean density within the tidal radius of each satellite. For clarity, the density profiles corresponding to the lower curves are vertically shifted downward by a factor of 0.05 . The $(\alpha, \beta, \gamma)$ density law provides an accurate description of the internal structure of cosmological subhalos at all radii, while the NFW functional form substantially underestimates subhalo densities in the innermost regions. Bottom panel: residuals for the density profile fits, $\Delta \rho / \rho \equiv \rho_{\text {sub }}-\rho_{\text {fit }} / \rho_{\text {sub }}$, where $\rho_{\text {sub }}$ is the true subhalo density computed in the cosmological simulation and $\rho_{\text {fit }}$ denotes the fitted density.

(A color version of this figure is available in the online journal.)

varying the other parameters. Moreover, we truncate subhalo profiles beyond the tidal radius with an exponential law

$$
\rho(r)=\rho\left(r_{\mathrm{tid}}\right)\left(\frac{r}{r_{\mathrm{tid}}}\right)^{\kappa} \exp \left(-\frac{r-r_{\mathrm{tid}}}{r_{\text {decay }}}\right) \quad\left(r>r_{\mathrm{tid}}\right),
$$

where $\kappa$ is fixed by the requirement that $d \ln \rho(r) / d \ln r$ is continuous at $r_{\text {tid }}$. This procedure is necessary because sharp truncations result in subhalo models that are not in equilibrium (Kazantzidis et al. 2004a), but it results in additional bound mass beyond $r_{\text {tid }}$. The precise amount of additional mass depends upon the model parameters, but is roughly $\sim 2 \%$ of the bound mass for each subhalo that we consider.

Figure 2 demonstrates the efficacy of our procedure for modeling the internal structure of cosmological subhalos. This figure presents spherically averaged density profiles, $\rho(r)$, of two representative cosmological satellites along with two different fits to their density distributions and the associated residuals, $\Delta \rho / \rho$. The first is a fit to the multiparameter $(\alpha, \beta, \gamma)$ functional form introduced above (Equation (1)), while the second is a fit to the NFW density profile with $(\alpha, \beta, \gamma)=$ $(1,3,1)$.

Figure 2 shows that $(\alpha, \beta, \gamma)$ models provide accurate representations of the density structures of cosmological subhalos.
This is an improvement over an NFW fit that is driven by additional parameter freedom. Indeed, the density residuals with respect to Equation (1) are $\lesssim 10 \%$ for both satellites over the entire range of radii, while the residuals to NFW fits are larger, reaching $\sim 50 \%$ in the innermost parts of the profile. This improvement is important because the dynamical response of galactic disks to satellite encounters depends upon the density distribution of the infalling systems, as we illustrate in Section 4.3 .

We list fit parameters for each subhalo in Table 1; however, we note two general features. First, in all cases the asymptotic outer slopes $\beta$ are found to be much steeper than 3 . Best-fit values vary from $\beta=3.7$ to $\beta=4.2$ suggesting that these satellites are better approximated by the Hernquist (1990) profile at large radii (see also Ghigna et al. 1998). Second, the intermediate slopes $\alpha$ range from $\alpha=0.1$ to 0.4 indicating a much more gradual transition between inner and outer asymptotic power laws compared to that of the NFW profile with $\alpha=1$. The steep outer profiles and variety in structural parameters is, at least in part, due to the strong dynamical evolution that subhalos experience within their host potential (e.g., Moore et al. 1996; Klypin et al. 1999a; Hayashi et al. 2003; Kazantzidis et al. 2004b; Kravtsov et al. 2004; Mayer et al. 2007).

$\mathrm{N}$-body realizations of satellites are constructed from a distribution function (DF) that self-consistently reproduces the density structures of selected subhalos S1-S6. Substructures in cosmological simulations are nearly spherical in both configuration and velocity space (Moore et al. 2004; Kazantzidis et al. 2006; Kuhlen et al. 2007), so we assume that the DF depends only upon the energy per unit mass and calculate it through an Abel transform (Binney \& Tremaine 1987; Kazantzidis et al. 2004a). We represent each satellite with $N_{\text {sub }}=10^{6}$ particles. The gravitational softening length is set to $\epsilon_{\text {sub }}=150 \mathrm{pc}$ which allows us to resolve the structure of subhalos to $\sim 1 \%$ of their tidal radii.

\subsubsection{Disk Galaxy Models}

We construct $N$-body realizations of multicomponent primary disk galaxies using the method of Widrow \& Dubinski (2005) as described in Paper I. The galaxy models consist of an exponential stellar disk, a Hernquist bulge (Hernquist 1990), and an NFW dark matter halo, and are characterized by 15 free parameters that may be tuned to fit a wide range of observational data for actual galaxies.

For the majority of satellite-disk encounter simulations, we use the specific parameter choices of model "MWb" in Widrow \& Dubinski (2005), which satisfies a broad range of observational constraints on the MW galaxy. The stellar disk has a mass of $M_{\text {disk }}=3.53 \times 10^{10} M_{\odot}$, a radial scale length of $R_{d}=2.82 \mathrm{kpc}$, and a sech ${ }^{2}$ scale height of $z_{d}=400 \mathrm{pc}$. The latter is consistent with that inferred for the old, thin stellar disk of the MW (e.g., Kent et al. 1991; Dehnen \& Binney 1998; Mendez \& Guzman 1998; Larsen \& Humphreys 2003; Jurić et al. 2008). It is reasonable to initialize the galactic disk with such thickness as observational evidence (e.g., Quillen \& Garnett 2000; Nordström et al. 2004) indicates that the scale height of the thin disk of the MW has not changed significantly since $z \approx 1$. The bulge has a mass and a scale radius of $M_{b}=1.18 \times 10^{10} M_{\odot}$ and $a_{b}=0.88 \mathrm{kpc}$, respectively. The dark matter halo has a tidal radius of $R_{h}=244.5 \mathrm{kpc}$, a mass of $M_{h}=7.35 \times 10^{11} M_{\odot}$, and a scale radius of $r_{h}=8.82 \mathrm{kpc}$. The total circular velocity of the galaxy model at the solar radius, $R_{\odot} \simeq 8 \mathrm{kpc}$, is $V_{c}\left(R_{\odot}\right)=234.1 \mathrm{~km} \mathrm{~s}^{-1}$ and the Toomre disk stability parameter is $Q=2.2$ at $R=2.5 R_{d}$. 
Direct numerical simulations of the evolution of model $\mathrm{MWb}$ in isolation for $10 \mathrm{Gyr}$ confirm its stability against bar formation. Therefore, any significant bar growth identified during the satellite-disk encounter experiments should be the result of subhalo bombardment. In what follows, we refer to this galaxy model as "D1."

In addition to our primary simulation set, we also address the dependence of disk response to encounters with infalling subhalos upon initial disk thickness and the presence of a bulge. Thicker disks have larger vertical velocity dispersions so we might expect them to be more robust to vertical heating by satellite accretion events. In addition, a central bulge component may act to reduce the amount of damage done to the structure of the inner disk by the infalling subhalos. Correspondingly, we initialize two additional disk galaxy models.

The first modified galaxy model was constructed with the same parameter set as D1, but with a scale height 2.5 times larger $\left(z_{d}=1 \mathrm{kpc}\right)$. We refer to this "thick" disk galaxy model as "D2." Except from disk thickness and vertical velocity dispersion, all of the other gross properties of the three galactic components of model D2 are within a few percent of the corresponding values for D1. The second modified galaxy model is the same as D1, but constructed without a bulge component. We refer to this "bulgeless" disk galaxy model as "D3." A bulgeless version of D1 constructed using the Widrow \& Dubinski (2005) method would differ significantly from D1. This is because the DF of the composite galaxy model is related to the individual density distributions and DFs of each galactic component in a nontrivial way. To mitigate such differences which make model comparison cumbersome, we realize model D3 by adiabatically evaporating the bulge from model D1 over a period of $500 \mathrm{Myr}$. During the evaporation process some properties of the inner disk and halo (e.g., density profiles, velocity ellipsoids) evolved in response to the decrease of the central potential; however, disk thickness remained largely unmodified. It is also worth mentioning that when evolved in isolation model D3 develops a bar inside $\sim 4 \mathrm{kpc}$ at time $t \sim 2 \mathrm{Gyr}$.

For each disk galaxy model, we generated an $N$-body realization containing $N_{d}=10^{6}$ particles in the disk and $N_{h}=2 \times 10^{6}$ particles in the dark matter halo. In experiments with models D1 and D2, bulges were represented with $N_{b}=5 \times 10^{5}$ particles. The gravitational softening lengths for the three components were set to $\epsilon_{d}=50 \mathrm{pc}, \epsilon_{h}=100 \mathrm{pc}$, and $\epsilon_{b}=50 \mathrm{pc}$, respectively. Mass and force resolution are sufficient to resolve the vertical structure of our galactic disks as well as minimize artificial heating of the disk particles through interactions with the much more massive halo particles. Lastly, in all cases we oriented the primary galaxy models such that the disk and host halo $\mathrm{G}_{1}$ angular momenta were aligned (e.g., Libeskind et al. 2007).

\subsection{Satellite-Disk Encounter Simulations}

All controlled simulations of satellite-disk interactions were carried out with the PKDGRAV code (Stadel 2001). We treated impacts of cosmological subhalos S1-S6 onto the disk as a sequence of encounters. Starting with subhalo S1 we included subsequent systems at the epoch when they were recorded in the cosmological simulation (Table 1). To limit computational cost, subhalos were removed from the controlled simulations once they reached their maximum distances from the disk after crossing, so satellites were not permitted to begin a second passage. In all cases, satellites lost $\gtrsim 80 \%$ of their mass after completing their first passage. This justifies our decision to neglect the dynamically insignificant subsequent crossing events.

The sequence of accretion events is such that S1 and S2 undergo simultaneous interactions with the disk. We have conducted an additional experiment in which satellite S2 was introduced only after the interaction with $\mathrm{S} 1$ was completed and confirmed that the disk dynamical response is similar in the two cases. When time intervals between subhalo passages were larger than the timescale needed for the disk to relax after the previous interaction, we introduced the next satellite immediately after the disk had settled from the previous encounter. This eliminates the computational expense of simulating the disk during the quiet intervals between interactions. The details of this procedure can be found in Paper I.

Due to their considerable mass and size, the infalling subhalos were not introduced in the simulations directly; instead, they were grown adiabatically in their orbits. This method ensures that the disk does not suffer substantial perturbations due to the sudden presence of the satellite and change in potential at its vicinity. Specifically, each encounter simulation was performed using the following procedure. (1) Insert a massless particle realization of each satellite at the distance at which it was recorded in the cosmological simulation of host halo $G_{1}$. (2) Increase the mass of this distribution to its final value linearly over a timescale that ranges between $\sim 150$ and $\sim 400 \mathrm{Myr}$ depending on subhalo mass. During this period, the satellite remained rigid and its particles are fixed in place, while the dark and baryonic components of the primary disk galaxy were allowed to achieve equilibrium with the subhalo as its mass grows. (iii) Initialize the "live" $N$-body satellite model by setting its internal kinematics according to the method described in Section 2.3.2, placing it on the desired orbit, and switching on its self-gravity. We note that after the satellite models are grown to full mass and have become self-gravitating, the tidal field of the primary disk galaxy acts to truncate their outer parts establishing new tidal radii that are smaller than the nominal values in Table 1. However, this difference is less than $10 \%$ in all cases suggesting that the primary disk galaxy model and the host CDM halo $\mathrm{G}_{1}$ have similar densities at $r_{\text {inf }}$.

We present results for the disk structure after allowing the disk to relax from the previous interaction. Consequently, our findings are relevant to systems that exhibit no obvious, ongoing encounters. Due to the complexity of the interaction, we determined this timescale empirically by monitoring basic properties of the disk structure (e.g., surface density, velocity dispersion, thickness) as a function of time. When these quantities stopped evolving significantly within radii of interest (changes of the order of 5\%-10\% in disk properties were considered acceptable), the encounter was deemed complete. Typical "settling" timescales were found to be in the range $\sim 500-600 \mathrm{Myr}$. The limiting radius was chosen to be $7 R_{\mathrm{d}}$ which contains $\sim 99 \%$ of the initial mass of the disk. It is necessary to adopt some limiting radius because dynamical times grow with radius and the outer disk regions continue to show signs of evolution well after each satellite passage. These transient structures can be readily identified in the edge-on views of the disk presented in Section 3.

We compute all disk properties and show all visualizations of the disk morphology after rotating the disk to the coordinate frame defined by the three principal axes of the total disk inertia tensor and centering it to its center of mass. The motivation behind performing these actions is twofold. First, infalling satellites tilt the disk through the transfer of angular momentum 


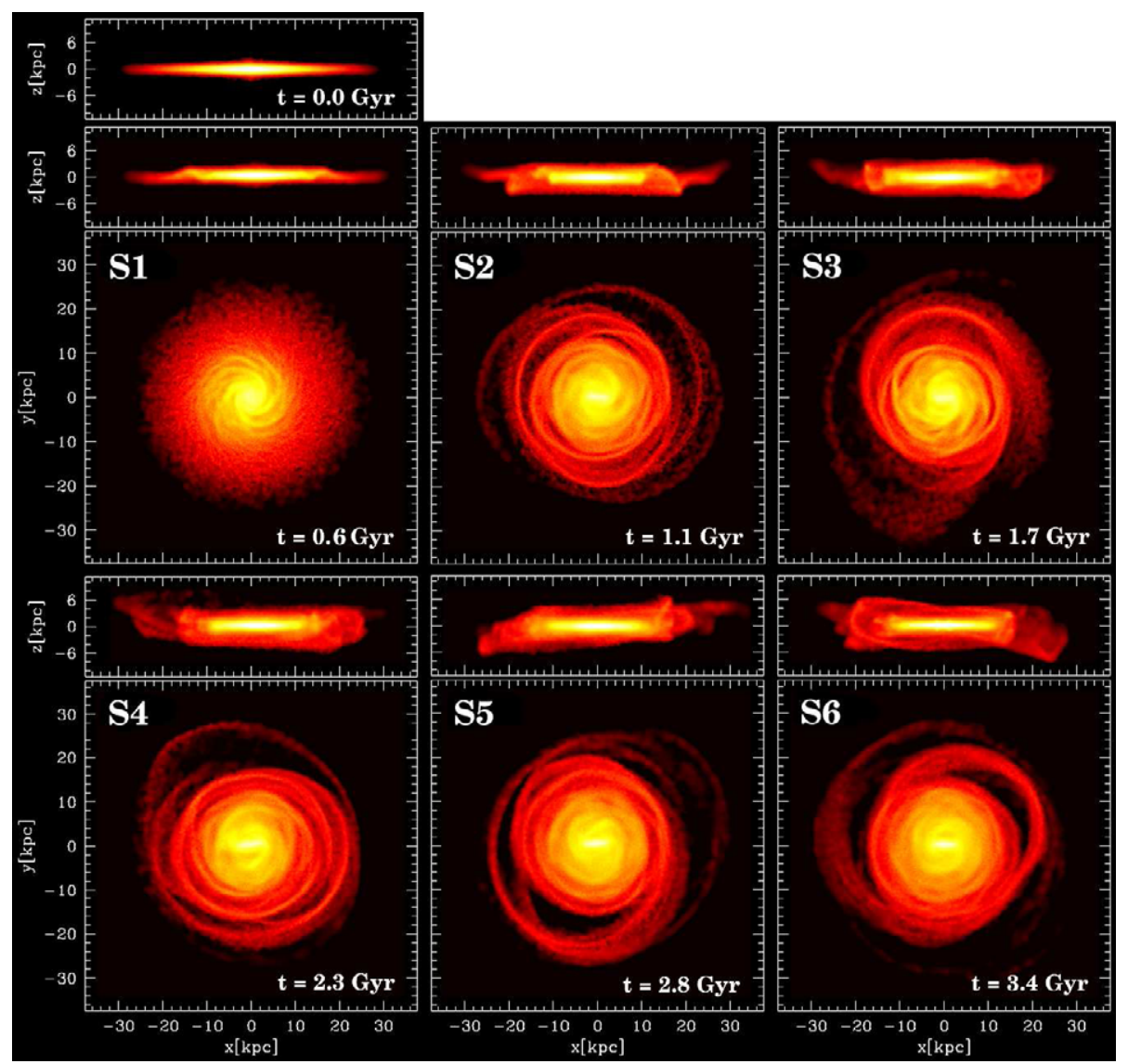

Figure 3. Density maps illustrating the global morphological evolution of the disk in galaxy model D1 subject to a typical $\Lambda$ CDM-motivated accretion history expected for a Galaxy-sized dark matter halo since $z \sim 1$. Particles are color-coded on a logarithmic scale with brighter colors indicating regions of higher stellar density. Local density is calculated using an SPH smoothing kernel of 32 particles. The face-on (bottom panels) and edge-on (upper panels) distributions of disk stars are shown in each frame and to aid comparison the first panel also includes the edge-on view of the initial disk. The labels for individual satellite passages from S1 to S6 and the time corresponding to each snapshot are indicated in the upper left-hand and lower right-hand corners of each bottom panel. Results are presented after centering the disk to its center of mass and rotating it to a new coordinate frame defined by the three principal axes of the total disk inertia tensor. The first satellite passage generates a conspicuous warp while the second encounter, which involves the most massive subhalo S2, causes substantial thickening of the disk and excites a moderately strong bar and extended ring-like features in the outskirts of the disk. Accretion histories of the kind expected in $\Lambda$ CDM models play a substantial role in setting the global structure of galactic disks and driving their morphological evolution.

(A color version of this figure is available in the online journal.)

(see Section 3.6). Introducing the new coordinate frame is important because in the original coordinate frame, rotation of stars in a tilted disk would appear as vertical motion interfering with the interpretation of the results. Second, the masses of the simulated satellites are a substantial fraction of the disk mass. As a result, the disk center of mass may drift from its initial position at the origin of the coordinate frame due to the encounters with the infalling subhalos.

\section{RESULTS: DYNAMICAL SIGNATURES OF HIERARCHICAL SATELLITE ACCRETION}

In this section, we examine the response of the thin, disk galaxy model D1 to interactions with cosmological subhalos S1-S6 of host halo $\mathrm{G}_{1}$, which are designed to mimic a typical central accretion history for a Galaxy-sized CDM halo over the past $\sim 8$ Gyr. While our simulation program focuses on halo $\mathrm{G}_{1}$, the similarity of subhalo populations in all four Galaxysized host halos suggests that the results presented next should be regarded as fairly general.

The "final" disk discussed in the next sections has experienced the S1-S6 encounters and was further evolved in isolation for $\sim 4.3 \mathrm{Gyr}$ after the last interaction, so that the disk evolution is followed from $z=1$ to $z=0$. The focus of this study is exclusively on the evolution of the disk material, so we do not consider the bulge component in any of the analysis presented below. We discuss in turn disk global morphology, thickening, velocity structure, surface density, lopsidedness, and tilting.

\subsection{Global Disk Morphology}

Figure 3 illustrates the global response of the disk to the infalling subhalos. The encounter with the first substructure (S1) generates a conspicuous warp beyond $\sim 12 \mathrm{kpc}$. The impact of the second most massive satellite (S2) has a dramatic effect on the global disk structure. The entire disk visually becomes considerably thicker compared to the initial model after this accretion event. In addition, this interaction excites extended ring-like features in the outskirts of the disk and a moderately strong bar (Paper I), both of which indicate that the axisymmetry of the initial disk is destroyed as a result of the first two accretion events. We stress that the bar is induced in response to the subhalo passages, not by amplified noise. It has a semimajor axis varying between $\sim 3$ and $4 \mathrm{kpc}$, within the range of values inferred for the bar in the MW (e.g., Bissantz \& Gerhard 2002). Throughout its evolution the bar fails to form a boxy bulge and remains rather thin. We note that we evolved the disk galaxy in isolation for $\sim 5 \mathrm{Gyr}$ after the encounter with $\mathrm{S} 1$ and verified 

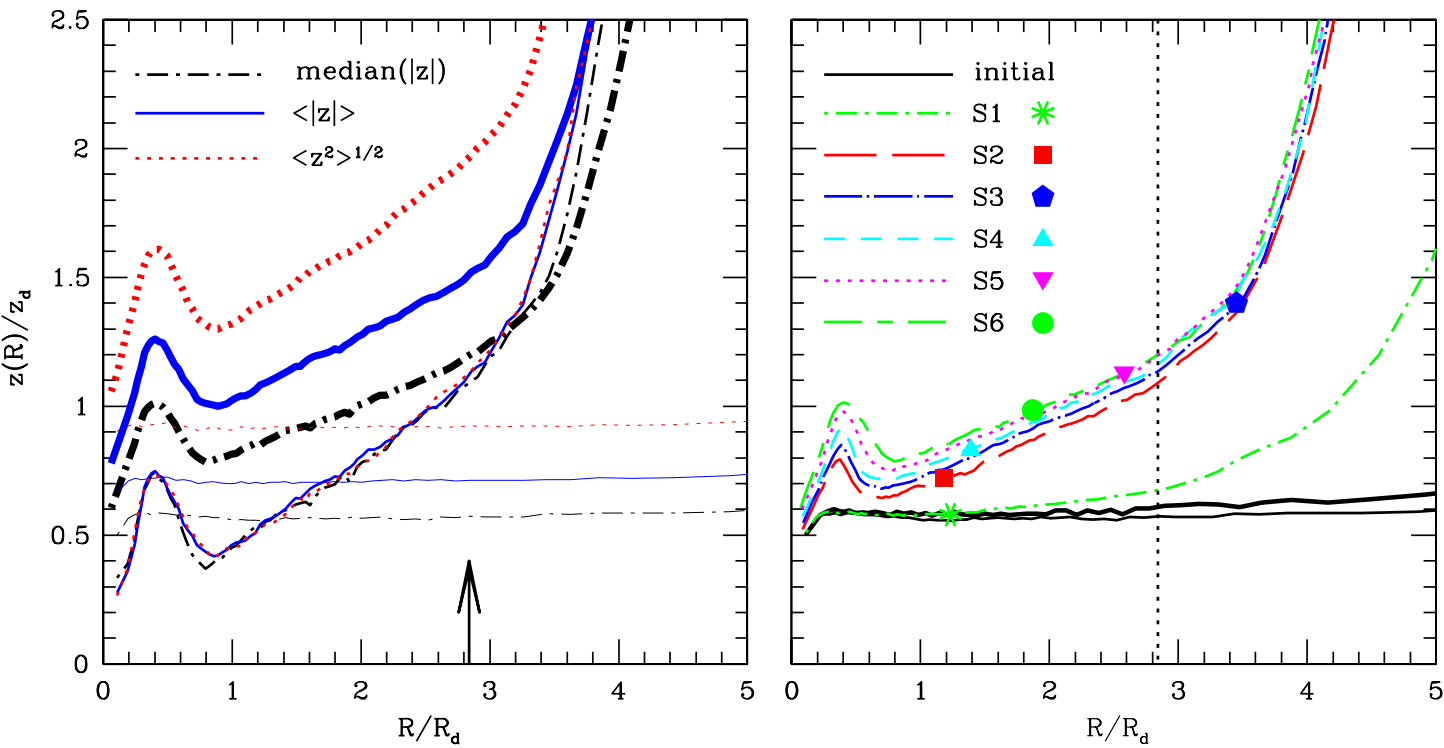

Figure 4. Disk thickening. Thickness profiles, $z(R)$, of the disk in galaxy model D1 viewed edge-on. Thicknesses and radii are normalized to the scale height, $z_{d}$, and radial scale length, $R_{d}$, of the initial disk. Left: thickness profiles for the initial (thin lines) and final disk (thick lines). Lines of intermediate thickness show the fractional increases in disk thickness defined as [median $(|z|)_{f}-$ median $\left.(|z|)_{i}\right] /$ median $(|z|)_{i}$, where median $(|z|)_{f}$ and median $(|z|)_{i}$ denote the thickness of the final and initial disk, respectively. Dot-dashed, solid, and dotted lines correspond to thicknesses measured by the median of the absolute value, median( $|z|)$, the mean of the absolute value, $\langle|z|\rangle$, and the dispersion, $\left\langle z^{2}\right\rangle^{1 / 2}$, of disk particle height above the midplane, respectively. The initial disk is constructed with a constant scale height, explaining why the corresponding curves are flat. The arrow indicates the location of the solar radius, $R_{\odot}$. The initial thin disk thickens considerably at all radii as a result of the encounters with CDM substructure and a conspicuous flare is evident in the final disk beyond $R \gtrsim 4 R_{d}$. Right: evolution of disk thickness profiles. Different lines show results for individual satellite passages from S1 to S6 and thicknesses are measured as median( $|z|)$. The vertical dotted line indicates $R_{\odot}$ and various symbols correspond to the pericenters of the infalling subhalos. The thick solid line corresponds to the initial disk evolved in isolation for a timescale equal to that of the combined satellite passages. The first two satellite passages thicken the disk considerably at all radii and cause substantial flaring in the outskirts. The combined effect of the remaining subhalos (S3-S6) is much less dramatic, indicating that the second, most massive accretion event is responsible for setting the scale height of the final disk.

(A color version of this figure is available in the online journal.)

that the first satellite alone is not capable of exciting a bar on these timescales. Fourier decomposition of the final disk also reveals that the radial variation of the amplitude of the observed spiral structure is similar to that of normal spiral galaxies in the near-infrared (e.g., Grosbol \& Patsis 1998).

Nonaxisymmetric structures such as the bar and the rings drive continued evolution by redistributing mass and angular momentum in the disk (e.g., Debattista et al. 2006). Thus, infalling satellites affect galactic disks not only directly by impulsively shocking the orbits of disk stars, but also indirectly by exciting global instabilities. In fact, angular momentum transport from one part of the disk to another causes the disk to expand radially during the encounters (see Section 3.4). However, this expansion should be significantly less pronounced compared to that in the vertical direction as rotational energy dominates over random motions in the plane of the disk.

Apart from changes associated with the structural details of the bar and the location and extent of the ring-like features, the disk structure does not evolve appreciably during subsequent satellite passages (S3-S6). In broad terms, the global morphological evolution of the galactic disk is driven by the interaction with the most massive subhalo of the accretion history. We will return to this point repeatedly in subsequent discussions. Lastly, the face-on projections of the disk show that the tidally induced bar and other structures survive long after the initial perturbations. Indeed, we confirmed their presence in the final disk some $\sim 4.3$ Gyr after the last encounter (see also Figure 6 in Paper I).

\subsection{Disk Thickening}

Among the most intriguing dynamical effects of the subhalo impacts is the pronounced increase in disk thickness, which is evident in the edge-on views of the disk in Figure 3. More quantitative measures disk thickening are the disk thickness profiles of Figure 4. The thickening of the disk may be described by the increase of its vertical scale height. We quantify disk thickness at any given radius by the median of the absolute value of disk particle height above the disk midplane, median $(|z|)$. The motivation behind this choice is threefold. First, it permits direct comparison with earlier work because similar estimators of disk thickness have been employed by previous authors (e.g., Quinn et al. 1993; Walker et al. 1996; Velazquez \& White 1999; Gauthier et al. 2006). Second, the gravitational interaction between satellites and disks is capable of heating individual disk stars to large vertical excursions. Choosing the median value of the particle height mitigates the influence of distant outlier particles. Third, disk scale heights must be formally derived by means of fitting the particle distribution to an appropriate functional form (e.g., exponential or a sech ${ }^{2}$ law). In contrast, quantities such as median $(|z|)$ do not require a fit to any functional form and so they are unambiguous and require no assumptions for their interpretation.

The left panel of Figure 4 shows thickness profiles for the initial and final disks together with the fractional increase in thickness caused by the infalling satellites. For convenience, in Figure 4 we compare the median $(|z|)$ to two additional estimators of disk thickness used extensively in the literature, namely the mean of the absolute value, $\langle|z|\rangle$, and the dispersion, $\left\langle z^{2}\right\rangle^{1 / 2}$, of disk particle height above the midplane. All estimators yield nearly similar fractional increases in disk thickness and we address only median $(|z|)$ in the remainder of the paper. We note that the initial disk exhibits a small departure from a pure exponential profile near its center. This feature develops when 
constructing the composite DF and potential of the multicomponent galaxy model. As a result, the stellar disk is somewhat thinner at its center. This deviation is insignificant for our purposes because it is confined to a small fraction of a scale length, $\sim 0.2 R_{d}$.

The left panel of Figure 4 demonstrates that the initial, thin disk thickens considerably at all radii as a result of the interactions with subhalos S1-S6. More specifically, disk thickness near the solar radius has increased in excess by a factor of 2. Thickening does not occur uniformly as a function of radius suggesting that the inner and outer disk regions respond differently to the accretion events. The outer disk is much more susceptible to damage by the accreting satellites. Indeed, at $R=R_{d}$ the thickness increases by $\sim 50 \%$ compared to a factor of $\sim 3$ rise at $R \sim 4 R_{d}$. The larger binding energy of the inner exponential disk and the presence of a massive, central bulge ( $\left.M_{b} \simeq 0.3 M_{\text {disk }}\right)$ which deepens the central potential are likely responsible for the robustness of the inner disk. Given the fact that the infalling subhalos are spatially extended and the self-gravity of the disk grows weaker as a function of distance from the center, it is not unexpected that disk thickness should increase with radius. Indeed, by making the simplest assumption that the accreting satellites deposit their orbital energies evenly in the disk, it can be shown that the disk scale height increases as $\Delta z(R) \propto \Sigma_{d}^{-2}(R)$, where $\Sigma_{d}(R)$ is the disk surface density (Paper I). It is also worth emphasizing that a significant part of the evolution seen in the inner parts of the disk $\left(R \lesssim R_{d}\right)$, including the local peak in the thickness profiles at $R \simeq 0.5 R_{d}$, can be attributable to the tidally induced bar.

The right panel of Figure 4 presents the evolution of disk thickness caused by individual satellite passages S1-S6. Though the masses of satellites $\mathrm{S} 1$ and $\mathrm{S} 2$ differ by less than a factor of 2 , subhalo $\mathrm{S} 2$ constitutes a far more efficient perturber compared to satellite S1. Taking into account that both subhalos have similar internal properties (Table 1) and are on approximately the same polar orbit, this suggests that disk thickening is very sensitive to perturber mass. In fact, using $N$-body simulations of satellite-disk encounters Hayashi \& Chiba (2006) found that the increase of the disk vertical scale height, $\Delta z_{d}$, is proportional to the square of the subhalo mass, $\Delta z_{d} / R_{d} \propto M_{\text {sub }}^{2}$. Our results suggest that this dependence may be even stronger.

The remaining satellite passages have a markedly less dramatic effect in perturbing the vertical structure of the disk. In part, this confirms that subhalo interactions with thicker disks induce smaller relative changes in disk thickness (Quinn et al. 1993; Paper I). We return to this point in Section 4.1. The combined effect of subhalos S3-S6 acts to increases the disk thickness at intermediate $\left(R_{\odot}\right)$ and large $\left(R=4 R_{d}\right)$ radii by only $\sim 10 \%$ compared to that after passage S2. The larger differences observed at small radii $\left(R \lesssim 1.5 R_{d}\right)$ are attributable to the bar, which grows stronger as a function of time. Finally, we remark that the thickness of the same disk galaxy evolved in isolation for a timescale equal to that of all satellite passages grows by only $\sim 10 \%$ indicating the excellent quality of the initial conditions and adequate resolution of the simulations.

\subsection{Disk Velocity Structure and Heating}

The dynamical response of a galactic disk to infalling satellites manifests itself in a variety of ways. In this section, we address the degree to which the disk velocity structure evolves as a result of the simulated accretion history. We characterize the disk kinematical response to accretion events through the evolution of the disk velocity ellipsoid $\left(\sigma_{R}, \sigma_{\phi}, \sigma_{z}\right)$, where $\sigma_{R}, \sigma_{\phi}$,

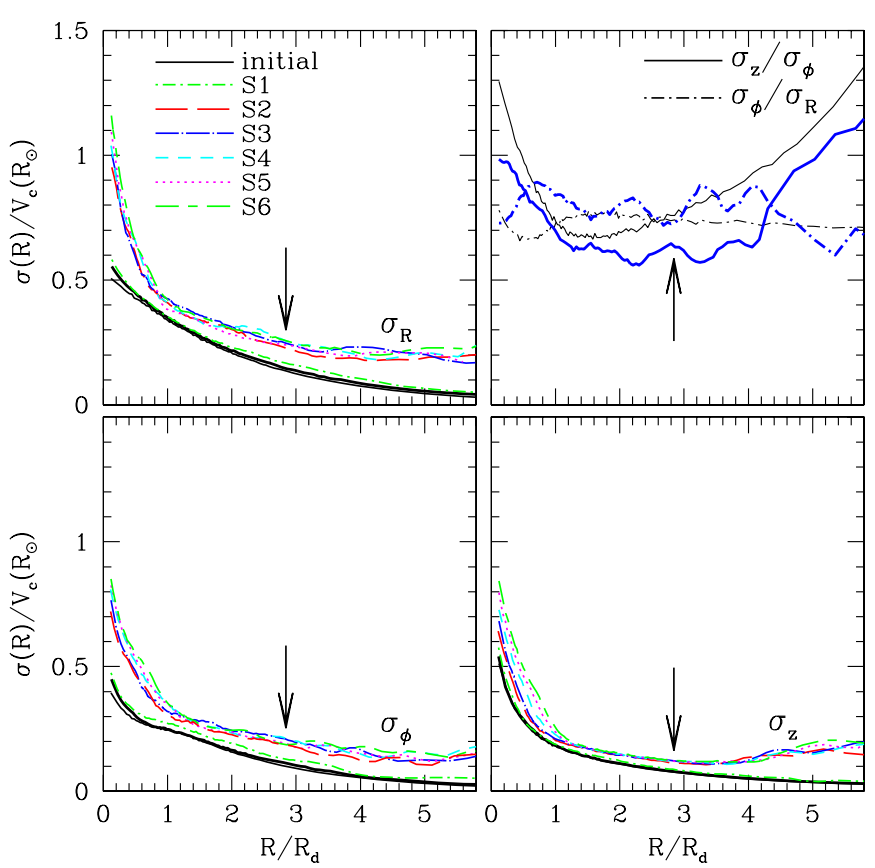

Figure 5. Disk heating. Velocity dispersion profiles of the disk in galaxy model D1 as a function of projected radius in units of the radial scale length of the initial disk, $R_{d}$. Counterclockwise from the upper left, the panels display the evolution of the radial, $\sigma_{R}$, azimuthal, $\sigma_{\phi}$, and vertical, $\sigma_{z}$, velocity dispersions. All profiles are normalized to the total circular velocity of galaxy model D1 at the solar radius, $V_{\mathrm{c}}\left(R_{\odot}\right)=234.1 \mathrm{~km} \mathrm{~s}^{-1}$. Different lines correspond to individual satellite passages from S1 to S6 and line types are the same as in the right panel of Figure 4. The thick lines present the kinematical properties of the primary disk galaxy evolved in isolation for a timescale equal to that of the combined satellite passages. In the upper right panel, the velocity dispersion ratios $\sigma_{z} / \sigma_{\phi}$ (solid lines) and $\sigma_{\phi} / \sigma_{R}$ (dot-dashed lines) are shown for the initial (thin lines) and final (thick lines) disk. In all panels, the arrows indicate the location of the solar radius, $R_{\odot}$. Bombardment by $\mathrm{CDM}$ substructure heats the thin galactic disk considerably in all three directions and causes its velocity ellipsoid to become more anisotropic.

(A color version of this figure is available in the online journal.)

and $\sigma_{z}$ correspond to the radial, azimuthal, and vertical velocity dispersions, respectively. In the isothermal sheet approximation, we expect that $\sigma_{z}^{2} \propto z_{d}$, and so the evolution of the disk vertical velocity dispersion should be less pronounced than the evolution of disk thickness.

Figure 5 reveals that the disk velocity ellipsoid grows significantly in all three directions as a result of the gravitational interactions with the infalling satellites. The disk velocity ellipsoid at the solar radius $R_{\odot}$ increases from $\left(\sigma_{R}, \sigma_{\phi}, \sigma_{z}\right)=$ $(31,24,17) \mathrm{km} \mathrm{s}^{-1}$ to $\left(\sigma_{R}, \sigma_{\phi}, \sigma_{z}\right) \simeq(61,49,31) \mathrm{km} \mathrm{s}^{-1}$. For reference, the velocity ellipsoid of the thick disk of the MW at $R_{\odot}$ is estimated to be $\sim(46,50,35) \mathrm{km} \mathrm{s}^{-1}$ by Chiba \& Beers $(2000)$ and $\sim(63,39,39) \mathrm{km} \mathrm{s}^{-1}$ by Soubiran et al. (2003). The velocity structure of the disk evolved in isolation for an equivalent period shows very small evolution. As with disk thickening, the velocity structure is influenced most dramatically by S2. Both the direct deposition of energy by the subhalos and global instabilities such as the bar and spiral structure which are excited during these encounters are responsible for the observed evolution. The latter phenomena act predominantly in the plane of the disk and cause the planar components of the disk velocity ellipsoid to evolve significantly.

Subhalo impacts heat the galactic disk in a nonuniform way. At small radii $\left(R \lesssim R_{d}\right)$, the planar velocity dispersions exhibit a steep increase which is again primarily a consequence of the 


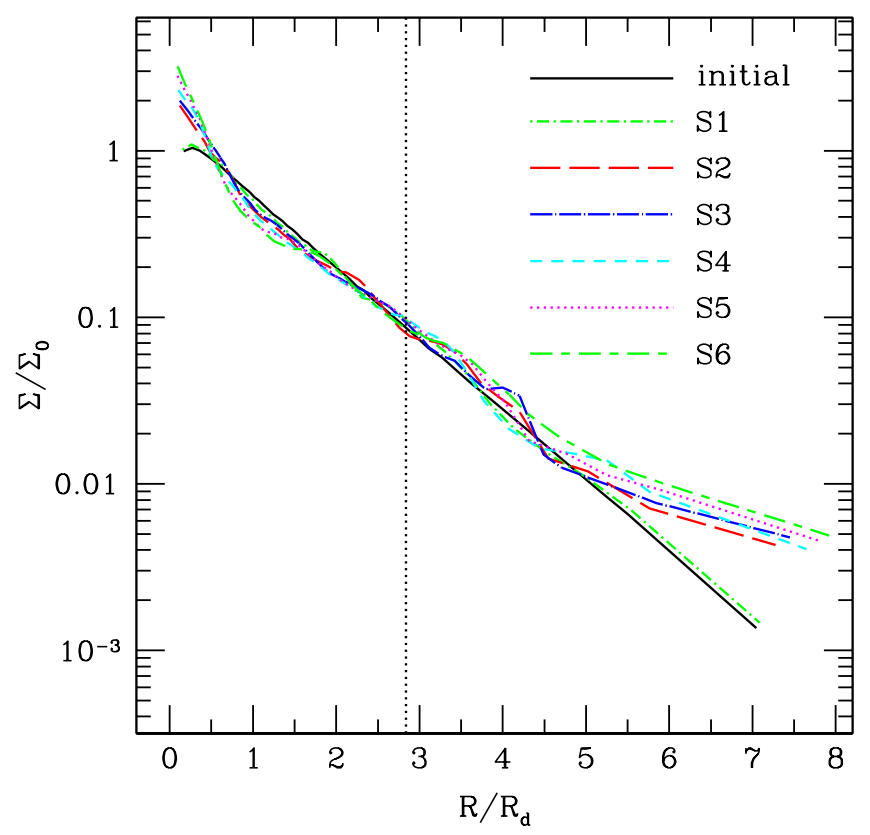

Figure 6. Disk antitruncation. Evolution of the surface density profiles, $\Sigma(R)$, of the disk in galaxy model D1 viewed face-on as a function of projected radius, $R$, in units of the radial scale length of the initial disk, $R_{d}$. Different lines show results for both the disk initially (solid line) and the disk after each subhalo impact as indicated in the upper right-hand corner. All profiles are normalized to the surface density of the initial disk at the origin, $\Sigma_{0}$, and the vertical dotted line indicates the location of the solar radius, $R_{\odot}$. Encounters with CDM substructure can generate surface density excesses in galactic disks similar to those seen in the light profiles of observed antitruncated disk galaxies.

(A color version of this figure is available in the online journal.)

bar. The upper right panel of Figure 5 shows that the ratio of $\sigma_{z}$ to $\sigma_{\phi}$ decreases at all radii as a result of the subhalo impacts. This finding, in conjunction with the fact that $\sigma_{\phi} / \sigma_{R}$ appears not to be affected in any noteworthy way, indicates that both planar components of the disk velocity ellipsoid respond more strongly to the accretion events compared to the vertical component. In other words, infalling satellites cause the disk velocity ellipsoid to become more anisotropic. It is worth noting that though the resultant heating is substantially larger in the plane, the disk spreads primarily in the vertical direction because rotational energy dominates over random motions in the plane of the disk.

Lastly, while the initial disk is designed so that its velocity ellipsoid decreases monotonically as a function of projected radius, after the satellite passages all dispersions become nearly constant outside $\sim 3 R_{d}$. Such flat velocity dispersion profiles are in very good agreement with results from recent kinematic studies of planetary nebulae in the extreme outskirts of spiral galaxies (Herrmann et al. 2009). In addition, though the initial dispersion profiles are smooth, at radii $R \gtrsim R_{d}$, the planar components $\sigma_{R}$ and $\sigma_{\phi}$ of the velocity ellipsoid exhibit wavelike features associated with the stellar rings in the disk plane seen in Figure 3.

\subsection{Disk Surface Density and Antitruncation}

In is interesting to investigate how the simulated subhalo accretion history would influence the disk surface density distribution. Figure 6 shows the evolution of the face-on surface density profile of the disk as a function of satellite passages S1-S6. By construction, the surface density profile of the initial disk follows an exponential distribution in cylindrical radius $R$.

As with both disk thickness and velocity structure, the disk surface density is minimally affected by the encounter with the first satellite $\mathrm{S} 1$. The response of the surface density distribution to subsequent accretion events is notable. At small radii $(R \lesssim$ $R_{d}$ ), the surface density profile steepens considerably, a feature that is again due to the bar and the transport of disk material inward by the spiral patterns and ring-like features. The central disk surface brightness increases by $\Delta \mu=1 \mathrm{mag} \operatorname{arcsec}^{-2}$ during the course of the interactions with subhalos S1-S6. At intermediate radii ( $R_{d} \lesssim R \lesssim 5 R_{d}$ ), the surface density profiles are very similar to that of the initial disk. Additionally, these profiles are not smooth but display strong wave-like features which are associated with the rings of disk material (Figure 3).

More interestingly, the disk spreads radially, and beyond $R \gtrsim$ $5 R_{d}$, there is a clear excess of surface density compared to that of the initial disk. The subhalo encounters modify the face-on structure of a single-component, exponential disk to producing a system with two distinct components. Fitting an exponential profile to the surface density of the final disk at $R_{d} \leqslant R \leqslant 5 R_{d}$ yields a best-fit scale length of $R_{d \text {,inner }} \approx 3.05 \mathrm{kpc}$, or $\sim 8 \%$ larger than the initial scale length. Fitting another exponential profile to the outer final disk $\left(R>5 R_{d}\right)$ yields a scale length of $R_{d \text {, outer }} \approx 5 \mathrm{kpc} \sim 1.8 R_{d} \sim 1.6 R_{d \text {,inner. }}$ This excess surface density at large radii relative to the inner exponential profile is interesting in the context of the so-called "antitruncated" stellar disks whose surface brightness profiles display similar excesses at roughly 4-6 disk scale lengths from galaxy centers (e.g., Erwin et al. 2005, 2008; Pohlen \& Trujillo 2006; Pohlen et al. 2007).

Our simulations lack the physical effect of star formation, so this disk antitruncation arises from old stars that resided in the initial thin disk and migrated outward. The driver behind the expansion of the disk and the excess surface density is redistribution of disk angular momentum (and hence disk mass) caused both directly and indirectly by the subhalo impacts. Infalling satellites directly deposit kinetic energy and angular momentum into the disk during the gravitational interactions. Additionally, nonaxisymmetric features such as the bar and spiral structure transport angular momentum and stellar mass to large radii. In response to the net transfer of angular momentum between its inner and outer regions, the disk expands. Indeed, we find that the magnitude of the total angular momentum at $R \gtrsim 5 R_{d}$ grows by $\sim 80 \%$ over the course of the simulated satellite accretion history. The transport of disk material outward in radius also leads to the excess surface density observed at large projected radii.

\subsection{Disk Lopsidedness}

An intriguing phenomenon observed in many disk galaxies is the existence of significant lopsided asymmetries in their neutral hydrogen and/or stellar mass distributions (e.g., Baldwin et al. 1980; Richter \& Sancisi 1994; Rix \& Zaritsky 1995; Zaritsky \& Rix 1997; Matthews et al. 1998; Haynes et al. 1998; Bournaud et al. 2005; Reichard et al. 2008). Specifically, the fraction of lopsided stellar disks varies between $\sim 20 \%$ and $\sim 30 \%$ for strongly lopsided systems and may exceed $50 \%$ for moderately lopsided disks (e.g., Rix \& Zaritsky 1995; Zaritsky \& Rix 1997; Rudnick \& Rix 1998; Bournaud et al. 2005). The face-on views of the simulated disk in Figure 3 illustrate that satellite encounters generate large-scale, long-lived asymmetries.

In what follows, we quantify the degree to which the simulated disk exhibits deviations from axisymmetry using the Fourier decomposition analysis. We express the surface 


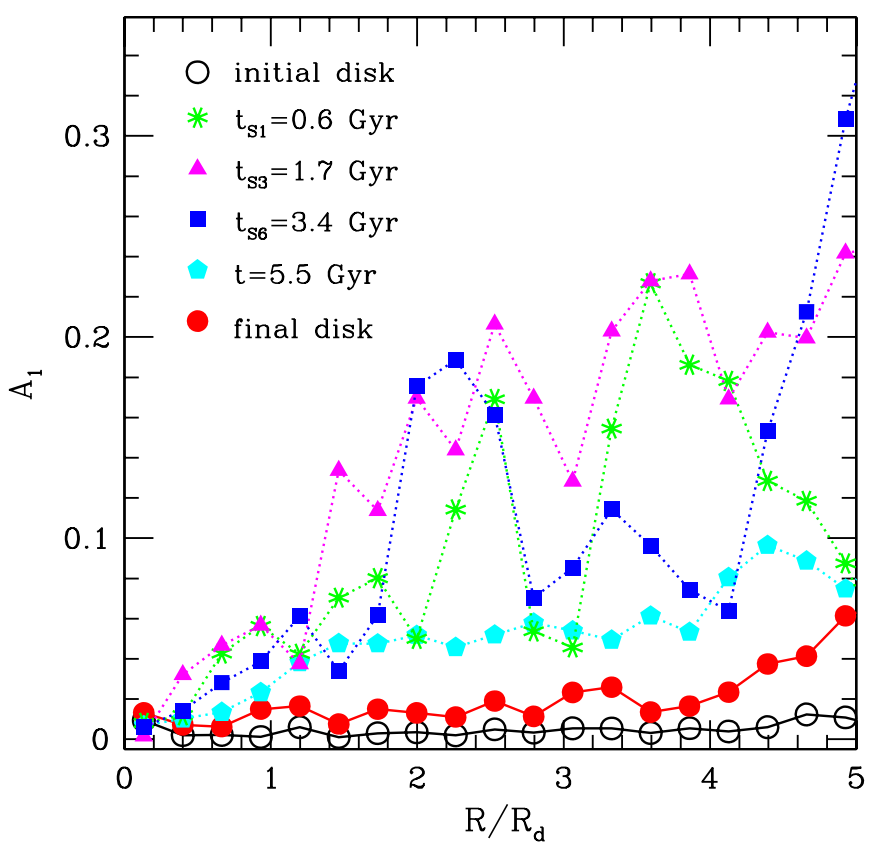

Figure 7. Disk lopsidedness. Evolution of the $A_{1}$ parameter, the normalized amplitude of the $m=1$ Fourier component of the disk surface density, of the disk in galaxy model D1 as a function of projected radius in units of the radial scale length of the initial disk, $R_{d}$. Results are presented for both the initial (open circles) and final distribution of disk stars (filled circles), and for four characteristic times in the simulated accretion history of host halo $\mathrm{G}_{1}$ (after each of the S1, S3, and S6 encounters, and $\sim 2$ Gyr subsequent to the last accretion event). $\Lambda$ CDM-motivated satellite accretion histories are responsible for triggering as well as maintaining for a significant fraction of the cosmic time lopsidedness in stellar disks at levels similar to those in the observed galaxies.

(A color version of this figure is available in the online journal.)

density of the stellar disk as a Fourier series

$$
\Sigma(R, \phi)=a_{0}(R)+\sum_{m=1}^{\infty} a_{m}(R) e^{\mathrm{im}\left[\phi-\phi_{m}(R)\right]}
$$

where $A_{m}(R) \equiv a_{m}(R) / a_{0}(R)$ and $\phi_{m}(R)$ denote the normalized strength and the phase of the Fourier component $m$, and quantify lopsidedness by the ratio of the amplitudes of the $m=1$ to $m=0$ (azimuthally averaged surface density) Fourier coefficients, $A_{1}(R) \equiv a_{1}(R) / a_{0}(R)$ (Rix \& Zaritsky 1995). To derive the results below, we performed the decomposition after centering the system to the peak of the density distribution (Rix $\&$ Zaritsky 1995), but using the centroid gave similar results (Debattista \& Sellwood 2000).

Figure 7 shows the variation of the lopsidedness parameter $A_{1}$ as a function of projected radius from the center of the disk. Results are presented for both the initial and final distribution of disk stars and for four characteristic timescales in the simulated accretion history of halo $\mathrm{G}_{1}$. The initial disk is constructed to be axisymmetric explaining why the corresponding curve is flat. Figure 7 clearly demonstrates that encounters with CDM substructures trigger lopsidedness in the galactic disk. Furthermore, the induced lopsidedness is not constant as a function of radius nor are changes monotonic. Different regions of the disk may thus become lopsided to different degrees by the infalling satellites.

During the simulated accretion history, typical values of $A_{1}$ span the range $0.1 \lesssim A_{1} \lesssim 0.2\left(1.5 R_{d} \lesssim R \lesssim 4.5 R_{d}\right)$, but more prominent lopsided asymmetries reaching amplitudes of $\sim 0.3$ is observed at larger radii $\left(R \gtrsim 5 R_{d}\right)$. These values are consistent with observational estimates of lopsidedness from various samples of stellar disks (e.g., Rix \& Zaritsky 1995; Zaritsky \& Rix 1997; Rudnick \& Rix 1998; Bournaud et al. 2005). The outer disk regions are more susceptible to the tidal perturbations that generate these asymmetries, and because they are characterized by longer dynamical times, significant lopsidedness persists there. Though the bombardment by CDM substructure has ceased by $\sim 3.5 \mathrm{Gyr}$, lopsidedness of moderate amplitude is still imprinted in the stellar disk $\sim 2$ Gyr after the last accretion event. The final disk, $\sim 4.3 \mathrm{Gyr}$ after the last satellite impact, exhibits little lopsided asymmetry at $R \lesssim 4.5 R_{d}$ and very weak lopsidedness at larger radii. The lifetimes of the reported lopsided asymmetries $(\sim 1 \mathrm{Gyr})$ are in agreement with estimates from phase mixing and winding arguments (e.g., Baldwin et al. 1980; Rix \& Zaritsky 1995) and direct analysis of $N$-body simulations (e.g., Zaritsky \& Rix 1997; Bournaud et al. 2005; Mapelli et al. 2008).

The main implication of the results reported in Figure 7 is that the continuous accretion of satellites over a galaxy's lifetime constitutes a significant source of external perturbations that excite as well as maintain lopsidedness in stellar disks at observed levels. This might be important as many observational studies find no correlation between the presence of nearby companions and lopsidedness in disk galaxies (e.g., Zaritsky \& Rix 1997; Wilcots \& Prescott 2004; Bournaud et al. 2005).

\subsection{Disk Tilting}

As discussed earlier, angular momentum exchange between the satellites and the disk is expected to tilt the disk relative to its initial orientation. The left panel of Figure 8 presents edge-on density maps of the initial and final disk. The final disk is shown relative to both the initial coordinate frame and the frame defined by the principal axes of the total disk inertia tensor. The panel reveals that the simulated subhalo impacts cause a significant amount of disk tilting. For this particular accretion history, the angle by which the disk is tilted from its original plane is $\theta \sim 11^{\circ}$, implying a significant transfer of angular momentum from the infalling satellites to the host galactic disk. This indicates that not all of the orbital energy associated with the vertical motion of the subhalos is converted into random motions of disk stars causing disk thickening. When viewed in the original coordinate frame, the final disk appears thicker, which is simply a consequence of the fact that the disk is substantially tilted by the orbiting satellites. Before continuing, we note that in the absence of satellites there is some exchange of angular momentum between the disk and the dark matter halo of the primary galaxy. This exchange results in tilts of $\lesssim 1^{\circ}$ when the galaxy is evolved in isolation for a timescale equal to that of the combined subhalo passages.

The right panel of Figure 8 shows a scatter plot of the evolution of the angular position of disk pole, defined by the azimuthal and zenith angles $(\phi, \theta)$, relative to initial disk pole as a function of satellite passages S1-S6. These angles are computed considering all disk particles within $15 \mathrm{kpc}$ from the disk center, but we note that the results do not depend sensitively upon this cutoff. The combined action of the first two satellites $\mathrm{S} 1$ and $\mathrm{S} 2$ serves to tilt the galactic disk by a very small amount $\theta \lesssim 1.5$. Both subhalos are on nearly polar orbits (Table 1) and angular momentum transfer is minimal. In this case, significant orbital energy associated with the $z$ motion of the infalling satellites is converted into random vertical stellar motions causing thickening of the disk. The next four interactions involve subhalos on both prograde (S3, S5) and 

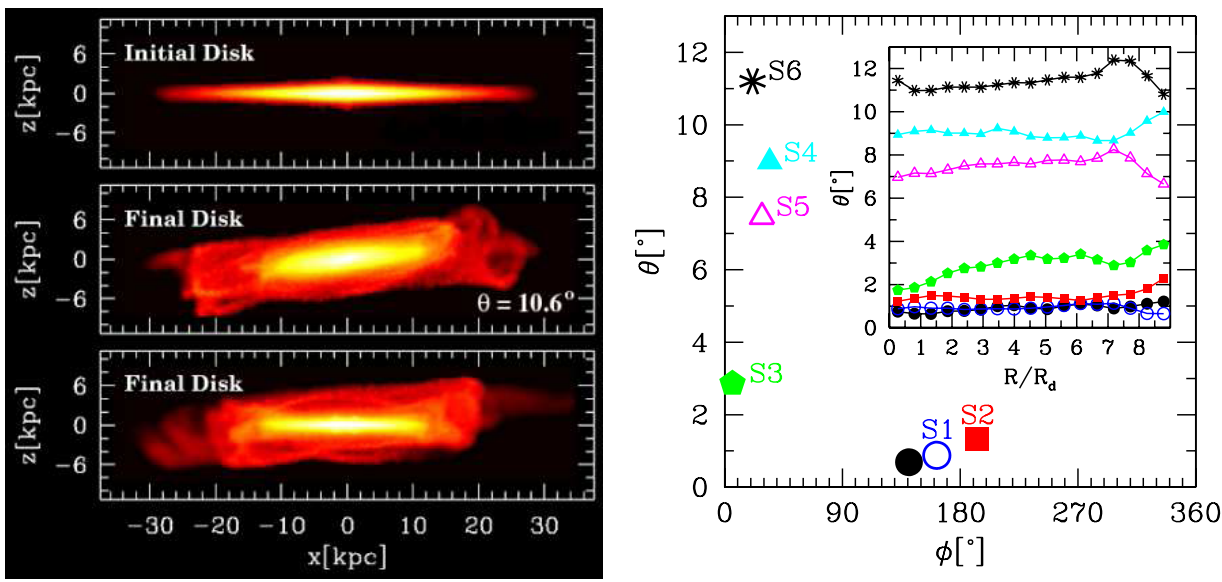

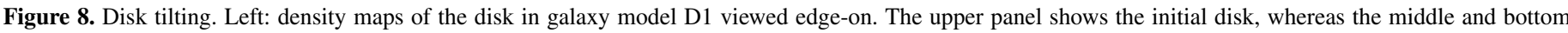

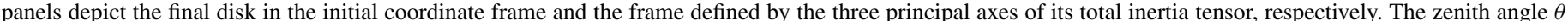

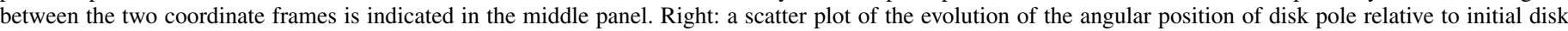

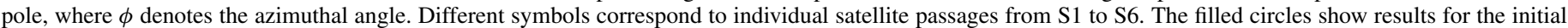

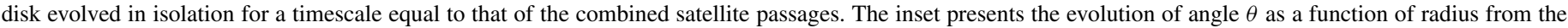

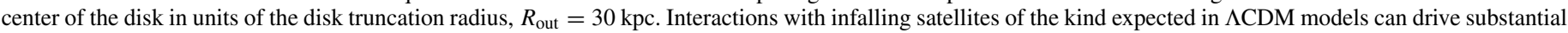
tilting in galactic disks.

(A color version of this figure is available in the online journal.)

retrograde (S4, S6) orbits, and efficient angular momentum exchange does occur. While these impacts result in $\theta \sim 11^{\circ}$, they keep $\phi$ between $0^{\circ}$ and $35^{\circ}$, indicating that each encounter induces a tilt about a similar axis. Lastly, the disk response to subhalo passages S3-S6 suggests that, in addition to the mass, the orbital orientation of the infalling satellite is crucial in determining the amount of disk tilting. Retrograde encounters appear to be associated with more pronounced tilting compared to their prograde counterparts. We address this issue explicitly in Section 4.4.

The inset in the right panel of Figure 8 presents the evolution of the angle $\theta$ between the original and tilted coordinate frames as a function of radius from the center of the disk. The maximum radius we consider for this calculation is $r=0.85 R_{\text {out }}=$ $25.5 \mathrm{kpc}$, where $R_{\text {out }}$ denotes the disk truncation radius (Widrow \& Dubinski 2005), which is set by the requirement that each radial bin should contain at least 1000 particles. This is an empirical criterion which ensures a robust determination of the inertia tensor in each bin. The inset illustrates that the angle $\theta$ between the original and tilted coordinate frames is not constant as a function of radius and changes are not monotonic. Different regions of the disk may thus be tilted to different degrees by the infalling satellites. Interestingly, the dense inner disk $\left(r / R_{\text {out }} \lesssim 0.5\right)$ responds nearly as a rigid body to the accretion events (see also Shen \& Sellwood 2006), while the outer disk shows more significant variation. While the tilting angles of the inner and outer parts of the disk are different from each other, which is indicative of the presence of warping, the maximum difference is relatively small $\left(\Delta \theta \lesssim 2^{\circ}\right)$. This justify our decision to employ the coordinate frame defined by the principal axes of the total inertia tensor of the tilted disk to compute disk properties rather than to adopt the appropriate local coordinate frame for each radius within the tilted disk.

\section{SENSITIVITY OF THE DISK DYNAMICAL RESPONSE TO MODELING CHOICES}

In this section, we explore various factors that could influence the dynamical response of galactic disks to infalling satellites. We discuss in turn initial disk thickness, the presence of a bulge component in the primary disk, the internal density distribution of the infalling systems, and the relative orientation of disk and satellite angular momenta.

\subsection{Initial Disk Thickness}

The results in Sections 3.2 and 3.3 suggest that subsequent subhalo encounters with already thickened disks produce smaller changes in disk thickness and disk velocity ellipsoid compared to the initial accretion events. In what follows we investigate the effect of initial disk thickness on the thickening and heating of a galactic disk by infalling satellites in a more controlled manner. For this reason we constructed a self-consistent disk galaxy identical to the standard thin-disk model but with a scale height that was larger by a factor of $2.5, z_{d}=1 \mathrm{kpc}$ (model D2). We then repeated the impacts of satellites S1 and $\mathrm{S} 2$ onto this thicker disk model.

Figure 9 shows the evolution of disk thickness as quantified by median $(|z|)$, and disk velocity ellipsoid $\left(\sigma_{R}, \sigma_{\phi}, \sigma_{z}\right)$, after each subhalo passage. There is little additional thickening in model D2 within roughly four scale lengths. Even beyond this radius, D2 is much less flared compared to disk model D1. More specifically, D2 thickens by less than $50 \mathrm{pc}$ at $R_{\odot}$, while the thickness of D1 increases by more than $200 \mathrm{pc}$ at the same radius. Likewise, we find that the velocity structure of the thicker initial disk is significantly less influenced by the perturbers. The velocity ellipsoid of model D2 increases by less than $\sim 15 \%$ at the solar radius due to the interactions with subhalos S1 and $\mathrm{S} 2$. The corresponding increase for galaxy model D1 is slightly less than a factor of 2 (Figure 5). It is also worth noting that model D2 does not form a bar as a result of the subhalo impacts and is associated with a much less prominent spiral structure compared to the thinner disk D1. These findings confirm that thicker disks exhibit a much weaker global dynamical response to accretion events compared to their thin counterparts.

\subsection{Presence of a Bulge Component}

The results presented in Figure 4 demonstrate that the inner disk regions exhibit substantial resilience to encounters with subhalos. Apart from the larger binding energy of the inner 

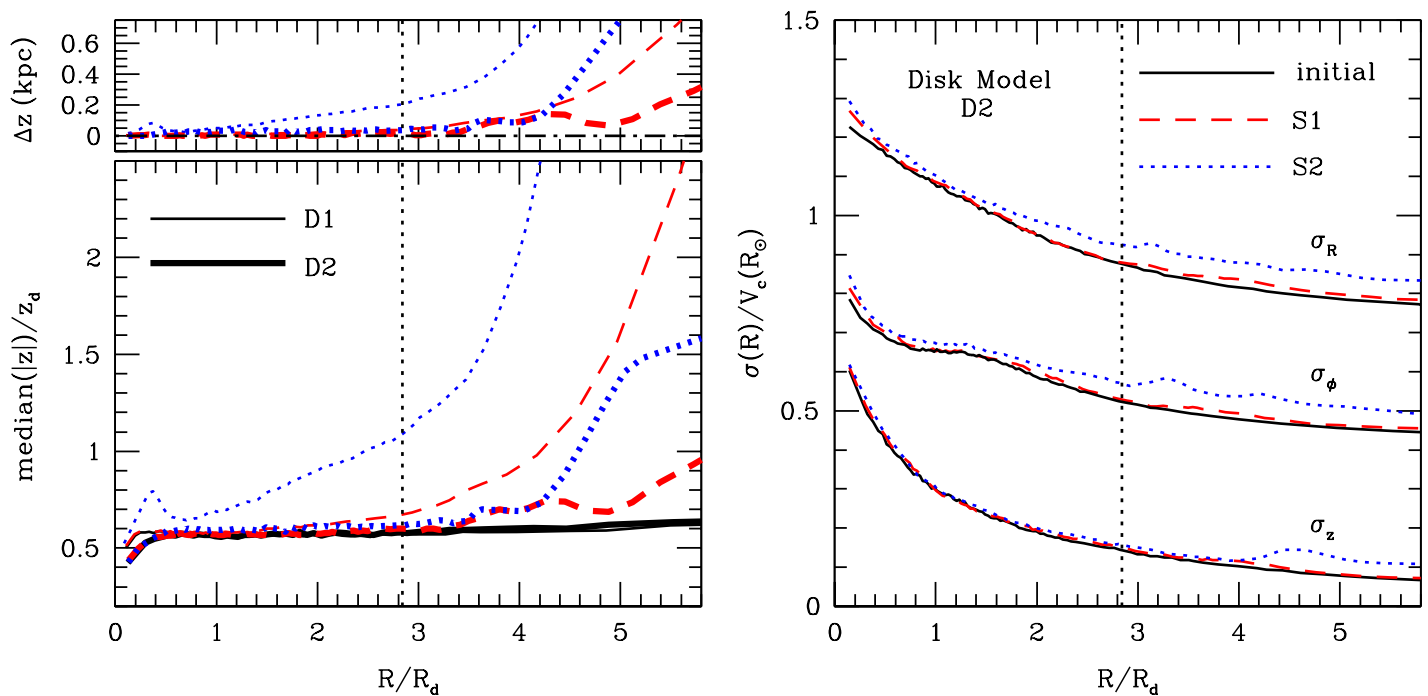

Figure 9. Effect of the initial disk thickness on the response of galactic disks to encounters with satellites. Bottom left panel: thickness profiles as a function of projected radius in units of the radial scale length of the initial disk, $R_{d}$. The thin lines show the evolution of thickness in the standard disk galaxy model D1 $\left(z_{d}=0.4 \mathrm{kpc}\right)$ after the interactions with subhalos S1 and S2. The thick lines present results for the same impacts onto the much thicker disk galaxy model D2 $\left(z_{d}=1 \mathrm{kpc}\right)$. The dashed and dotted lines correspond to satellites S1 and S2, respectively, while the solid curves show results for the initial disks. All curves are normalized to the initial scale height of each corresponding disk. Upper left panel: thickness increase, $\Delta z \equiv\left[\right.$ median $(|z|)_{\text {final }}-$ median $\left.(|z|)_{\text {initial }}\right]$. Here, median $(|z|)_{\text {initial }}$ and median $(|z|)_{\text {final }}$ denote the thicknesses of the disk initially and after each subhalo impact, respectively. Right: evolution of the disk velocity ellipsoid in galaxy model D2. Bottom to top: $\sigma_{z}$, $\sigma_{\phi}+100 \mathrm{~km} \mathrm{~s}^{-1}$, and $\sigma_{R}+175 \mathrm{~km} \mathrm{~s}^{-1}$. The line types are as in the left panel, but the results for galaxy model D1 are not shown. All profiles are normalized to the total circular velocity of model D2 at the solar radius, $V_{\mathrm{c}}\left(R_{\odot}\right)=235.6 \mathrm{~km} \mathrm{~s}^{-1}$. The vertical dotted lines in all panels indicate the location of the solar radius, $R_{\odot}$. Thicker disks are less susceptible to damage by infalling subhalos compared to their thin counterparts.

(A color version of this figure is available in the online journal.)

exponential disk, the presence of a massive, central bulge with $\left(M_{b} \simeq 0.3 M_{\text {disk }}\right)$ in disk model D1 may be responsible for the robustness of the inner disk. To address the effect of a bulge in reducing the damage induced in galactic disks by infalling satellites, we repeated encounters S1 and S2 after adiabatically evaporating the bulge component from disk model D1 (see Section 2.3.3). We refer to the resulting bulgeless disk galaxy model as D3.

Figure 10 shows the evolution of disk thickness after each subhalo passage for both galaxy models D1 and D3. Not surprisingly, this exercise demonstrates that a massive bulge enhances the robustness of galactic disks to accretion events. Analysis of the disk velocity ellipsoids supports this conclusion. As expected, the relative effect of the bulge grows weaker as a function of radius from the center of the disk. The combined action of satellites S1 and S2 increases the thickness of model D3 at $R_{\odot}$ by a factor of $\sim 2.6$, compared to a factor of $\sim 2$ in the case of model D1. At larger radii $\left(R \gtrsim 3.5 R_{d}\right)$, model D3 is characterized by a more distinct flare compared to D1. This is interesting as the bulge is located in the inner regions of the disk and suggests that global instabilities which are curtailed by a massive bulge are efficient at driving significant evolution in the outer disk. Indeed, we stress that subhalo passages S1 and S2 excite a much stronger bar in model D3 compared to D1 and that this bar already develops in response to the interaction with S1.

\subsection{Satellite Internal Density Distribution}

It is worthwhile to examine the importance of the satellite internal density distribution to the structural changes induced in galactic disks. This interest is motivated by the potential differences in subhalo structure in models with modified dark matter properties and density fluctuation spectra (e.g., Hogan \& Dalcanton 2000; Avila-Reese et al. 2001), or the presence of

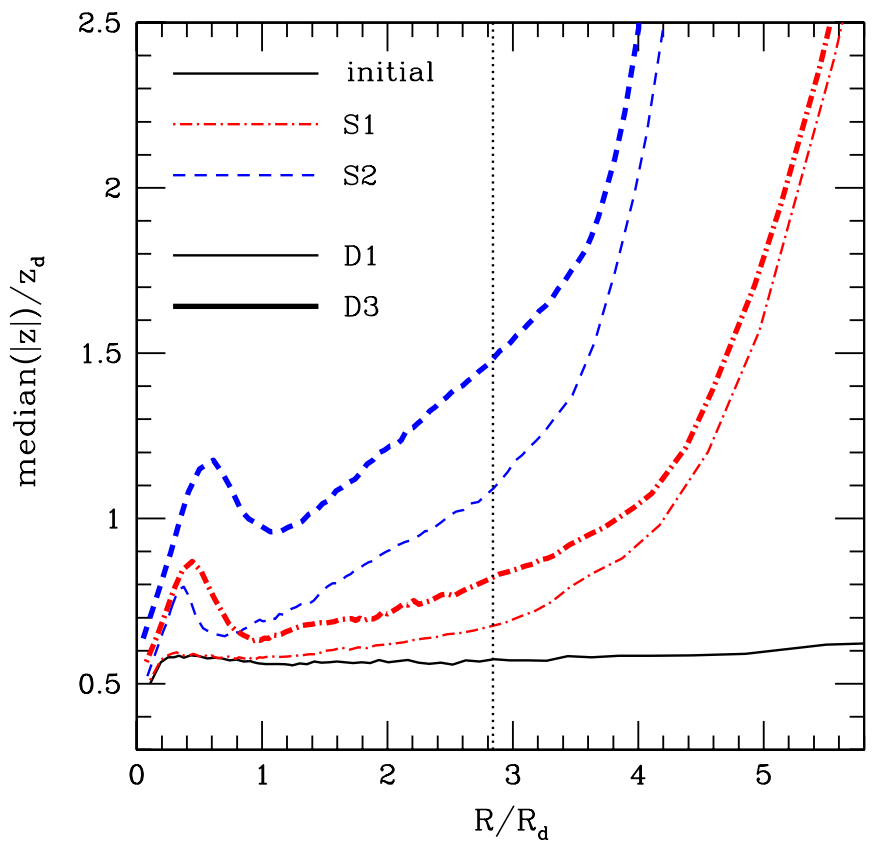

Figure 10. Effect of a bulge component on disk thickening. The thin lines show thickness profiles for disk galaxy model D1 after the encounters with subhalos $\mathrm{S} 1$ and S2. The thick lines present results for the same impacts onto the bulgeless disk model D3. Thicknesses and radii are normalized to the scale height, $z_{d}$, and radial scale length, $R_{d}$, of the initial disk. The dot-dashed and dashed lines correspond to satellites S1 and S2, respectively, while the solid curve shows results for the initial disk. A massive bulge enhances the resilience of galactic disks to satellite impacts by both absorbing part of the orbital energy deposited by the infalling subhalos and stabilizing the disks against the development of global nonaxisymmetric instabilities.

(A color version of this figure is available in the online journal.)

baryonic components in some fraction of the accreting systems. To this end, we repeated the encounters between disk galaxy 

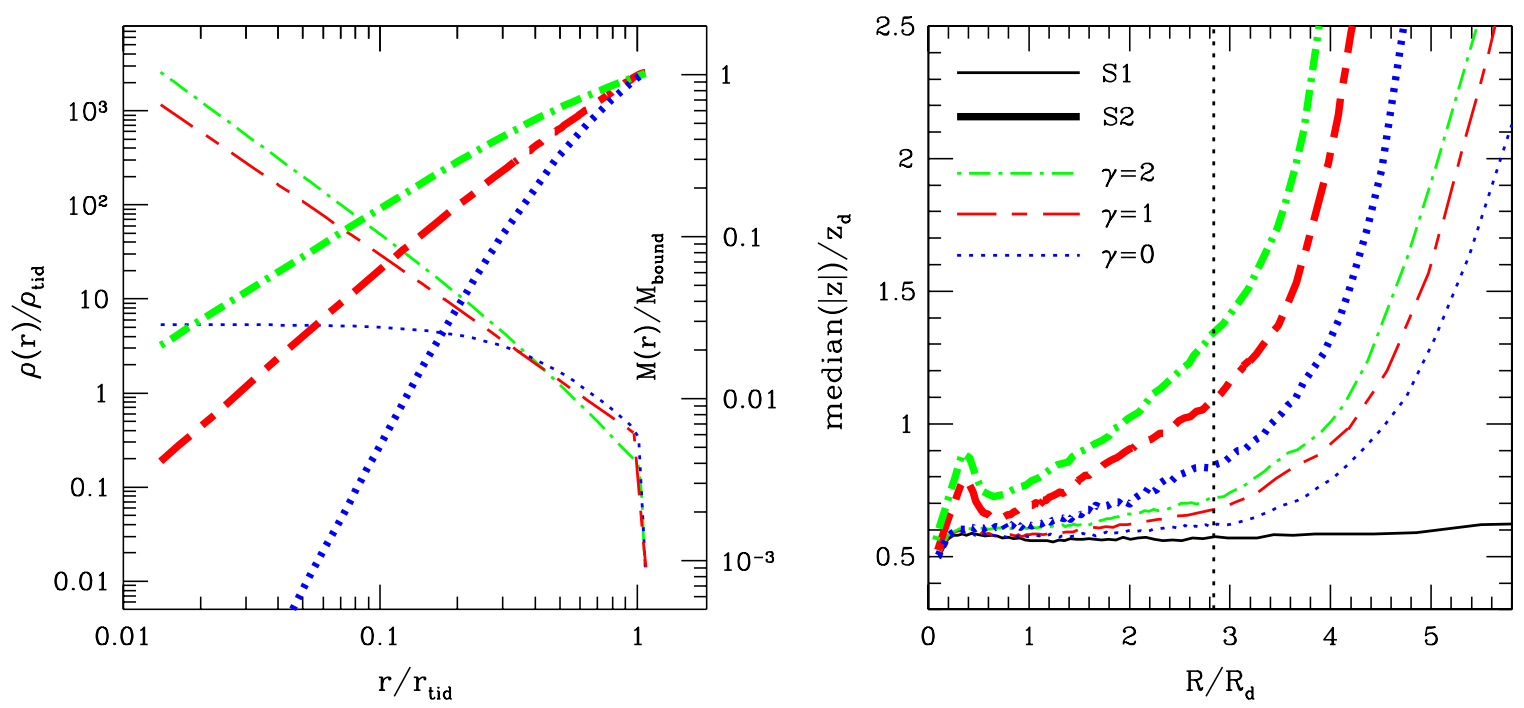

Figure 11. Effect of satellite internal density distribution on disk thickening. Left: spherically averaged density, $\rho(r)$, (thin lines) and cumulative mass profiles, $M(r)$, (thick lines) for infalling subhalos with different internal structure as a function of radius in units of the tidal radius, $r_{\text {tid. }}$. Densities and masses are normalized to the enclosed density within the tidal radius, $\rho_{\text {tid }} \equiv 3 M_{\text {bound }} / 4 \pi r_{\text {tid }}^{3}$ and the bound mass of the system, $M_{\text {bound }}$ (right axis), respectively. Results are presented for cosmological satellite S2. Density profiles differ only in the asymptotic inner slope $\gamma$ in Equation (1). The short dashed-long dashed lines show results for the standard density profile with $\gamma=1$, while the dotted and dot-dashed lines correspond to profiles with an inner power-law index equal to $\gamma=0$ and $\gamma=2$, respectively. The adopted density distributions have significantly different shapes, but they correspond to exactly the same bound mass. Right: evolution of disk thickness induced by satellites S1 and S2 following the density profiles described above. The thin and thick lines display results for the passages of satellites S1 and S2, respectively, and line types are the same as in the left panel. The solid line shows results for the initial disk model D1. Thicknesses and radii are normalized to the scale height, $z_{d}$, and radial scale length, $R_{d}$, of the initial disk. The vertical dotted line indicates the location of the solar radius, $R_{\odot}$. Infalling subhalos with steeper density distributions are more efficient perturbers compared to their cored counterparts.

(A color version of this figure is available in the online journal.)

model D1 and satellites S1 and S2 after assigning different density profiles to each infalling subhalo.

We employed density distributions that differ only in their asymptotic inner slopes, $\gamma$ (see Equation (1)) and required the total bound mass of each object to be fixed to its fiducial value. We studied two, relatively extreme options for the satellite density profiles with the intent that these bracket the range of potential subhalo structures. The first corresponds to a "steep" profile with an inner power-law index equal to $\gamma=2$, while the second follows a "shallow" profile with a constant density core, $\gamma=0$. The size of the density core was chosen to be approximately equal to $10 \%$ of the tidal radius of each satellite, $\gtrsim 2 \mathrm{kpc}$ (Table 1). The steep profile addresses the possibility of a subhalo containing a galaxy and which may have undergone adiabatic contraction in response to the growth of baryons (Blumenthal et al. 1986), while the cored profile is designed to overestimate any density suppression that may result in modified dark matter models. The density, $\rho(r)$, and cumulative mass profiles, $M(r)$, for all initial satellite models are presented in the left panel of Figure 11.

The right panel of Figure 11 presents the evolution of disk thickness in these alternative models compared with the standard cases. Not surprisingly, the amount of disk thickening increases with the steepness of the inner satellite density profiles at fixed initial subhalo mass. The combined action of cored satellites $\mathrm{S} 1$ and $\mathrm{S} 2$ increases the disk thickness at the solar radius by $\sim 45 \%$, compared to a factor of $\sim 2$ in the case of the standard satellites. The cumulative effect of the steep subhalos S1 and S2 is to increase the disk thickness by nearly a factor of $\sim 2.5$ at $R_{\odot}$. The relative thickness differences driven by the different density distributions becomes more pronounced at large radii where the disk self-gravity, and thus its restoring force, is weaker. It is worth noting that steep satellites S1 and S2 excite a stronger bar compared to their $\gamma=1$ counterparts, whereas cored satellites
S1 and S2 do not induce a bar at all on the timescales of these simulations. These findings indicate that satellite density structure as characterized by the inner slope or concentration is an important factor in determining the amount of damage and global dynamical evolution that subhalos impart upon disks. This is an important point in light of the fact that most previous numerical investigations of satellite-disk interactions did not realize their satellite models with the exact density structure of cosmological subhalos.

The primary reason for the dependence of disk dynamical evolution on satellite structure is that subhalos with shallower profiles are significantly less tightly bound. As a consequence, they generate much smaller potential fluctuations when they cross the disk and correspondingly cause less thickening and heating. In addition, cored satellites lose mass more efficiently even prior to the actual disk crossing. As a result, they penetrate the disk with less bound mass, and thus less energy and angular momentum available to be delivered to the disk stars. Owing to their lower self-gravity, the outer disk regions are expected to be more sensitive to the amount of orbital energy deposited by the infalling satellites. This is also confirmed by the findings presented in the right panel of Figure 11.

\subsection{Orientation of Satellite Orbits}

It is interesting to investigate any correlation between disk dynamical response and the orientation of satellite orbits. To this end, we repeated satellite passages $\mathrm{S} 1$ and $\mathrm{S} 2$ varying the angle $\theta$ between their orbital angular momentum and the initial angular momentum of the disk in galaxy model D1. We considered two cases with identical initial orbital inclinations with respect to the plane of the host disk $\left(i=45^{\circ}\right)$, but exactly opposite directions with respect to its rotation. The first is a prograde orbit with $\theta=45^{\circ}$ and the second is a retrograde orbit with $\theta=135^{\circ}$. 


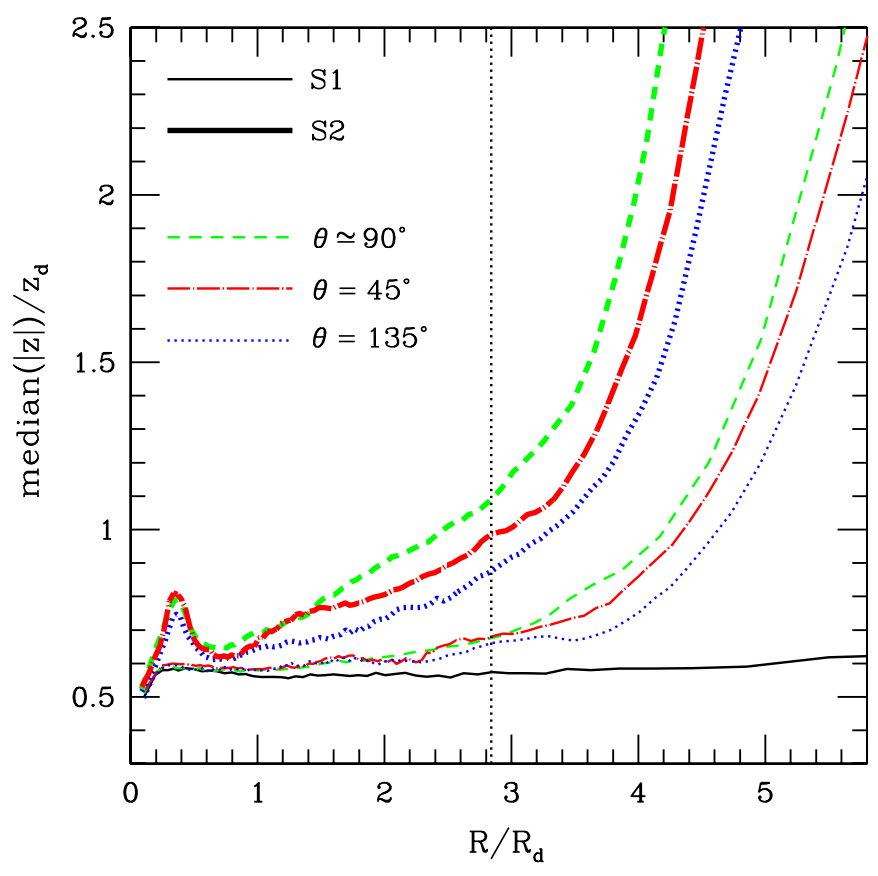

Figure 12. Effect of satellite orbital orientation on disk thickening. Evolution of disk thickness profiles for disk galaxy model D1 in response to encounters with subhalos $\mathrm{S} 1$ (thin lines) and S2 (thick lines). Thicknesses and radii are normalized to the scale height, $z_{d}$, and radial scale length, $R_{d}$, of the initial disk. The dashed lines correspond to our fiducial experiments with $\theta \simeq 90^{\circ}$. The dot-dashed and dotted lines show results for satellites S1 and S2 on a prograde $\left(\theta=45^{\circ}\right)$ and a retrograde orbit $\left(\theta=135^{\circ}\right)$, respectively. The solid line shows results for the initial disk model D1. The vertical dotted line indicates the location of the solar radius, $R_{\odot}$. Prograde encounters are more efficient at thickening the disk compared to their retrograde counterparts.

(A color version of this figure is available in the online journal.)

Figure 12 compares the results of these simulations with our fiducial experiments in which $\mathrm{S} 1$ and S2 are on nearly polar orbits with $\theta \simeq 90^{\circ}$ (Table 1 ). Encounters with subhalos on prograde orbits are more efficient at thickening the disk compared to their retrograde counterparts. This is suggestive of a resonant coupling between the satellite and disk angular momenta that is suppressed in retrograde encounters. After the completion of passage S2, differences in disk thickness between prograde and retrograde interactions manifest at all radii. Prograde satellites S1 and S2 increase the disk thickness by $\sim 20 \%$ more compared to the corresponding retrograde models. Moreover, retrograde $\mathrm{S} 1$ and $\mathrm{S} 2$ impacts excite a weaker bar than the prograde encounters.

Interestingly, neither prograde nor retrograde orbits cause as much thickening (or heating) as the nearly polar orbits of S1 and $\mathrm{S} 2$ in our fiducial simulations. However, the combined action of $\mathrm{S} 1$ and $\mathrm{S} 2$ tilts the disk by $\theta \sim 7.5$ in the prograde case and $\theta \sim 9.5$ in the retrograde case. This is to be compared to $\theta \lesssim 1.5$ in the fiducial case where S1 and S2 are on nearly polar orbits. This reinforces our interpretation in Section 3.6 of the different degrees of tilting among encounters S1-S6 as a manifestation of the different orbital orientations of the infalling satellites relative to the disk. During the encounters, part of the orbital energies and angular momenta of the infalling satellites are transferred into the disk. A fraction of this energy is converted into random vertical motions of disk stars, causing disk thickening, and a fraction is added to the disk coherently leading to its tilting. Because polar orbits do not transfer angular momentum, the fraction of the satellite orbital energy that thermalizes in the disk causing its thickening is larger. On the other hand, efficient exchange of angular momentum results in a large-scale, coherent tilting of the disk, and thus in smaller degrees of thickening, an effect that is most pronounced in the retrograde orbits.

We note that since we only consider interactions with satellites that each pass the disk only once, our numerical experiments cannot address the importance of satellite decay rate and mass loss in affecting any of the aforementioned trends. This is especially relevant in the case of retrograde and polar orbits which are known to suffer slower tidal disruption and orbital decay compared to their prograde counterparts (Velazquez \& White 1999).

\section{DISCUSSION}

We have demonstrated that encounters with halo substructure in the context of the $\Lambda \mathrm{CDM}$ cosmogony imprint a wealth of dynamical signatures in thin galactic disks. These signatures include considerable thickening and heating at all radii, surface density excesses resembling those of observed antitruncated disks, lopsidedness at levels similar to those measured in observational samples of disk galaxies, and significant tilting. In Paper I, we also showed that the same accretion events produce conspicuous flares, bars, low-surface brightness ringlike features in the outskirts of the disk, faint filamentary structures above the disk plane, and a complex vertical structure that is well described by a superposition of thin- and thick-disk components. All of these findings highlight the significant role of encounters with CDM substructure in setting the structure of disk galaxies and driving galaxy evolution.

In the present study, we have only utilized a conservative subset $\left(0.2 M_{\text {disk }} \lesssim M_{\text {sub }} \lesssim M_{\text {disk }}, r_{\text {peri }} \lesssim 20 \mathrm{kpc}\right)$ of a typical accretion history of a Galaxy-sized host halo to seed our controlled satellite-disk encounter simulations, and have neglected interactions with extremely massive subhalos $\left(M_{\text {sub }} \gtrsim\right.$ $M_{\text {disk }}$ ) that could prove ruinous to thin-disk survival (Purcell et al. 2009). In this sense, our results are relevant to systems that have already experienced the most destructive events since $z=1$ and have re-grown their thin disks since. Extending our selection criteria to include subhalos with smaller masses $\left(M_{\text {sub }} \lesssim 0.2 M_{\text {disk }}\right)$ and/or larger pericenters $\left(r_{\text {peri }} \gtrsim 20 \mathrm{kpc}\right)$ will definitely result in many more accretion events to consider. This will perhaps change the detailed results for the disk dynamical response subject to a $\Lambda \mathrm{CDM}$ accretion history, but it will not affect any of the qualitative conclusions of this study. If anything, the tidal effects of halo substructure will be more pronounced compared to that we reported here.

We also emphasize that our numerical experiments were not designed to elucidate the effect of subhalo bombardment on the structure of specific galaxies such as the MW or M31. Rather, the main goal of the present study was to investigate the most generic dynamical signatures induced in thin galactic disks by a typical $\Lambda$ CDM-motivated satellite accretion history. To this end, we utilized only four Galaxy-sized dark matter halos to extract representative orbits and subhalo merger histories, and the controlled simulations of satellite-disk interactions were based on the accretion history of just one of these host halos. Nevertheless, all four of these host halos showed similar numbers of substantial accretion events onto their central regions, and their accretion histories are typical of systems in this mass range (e.g., Wechsler et al. 2002). In addition, the bulk of the disk dynamical response illustrated in Figures 4 7 was driven by the single most massive substructure of the simulated accretion history $\left(M_{\text {sub }} \sim 0.6 M_{\text {disk }}, r_{\text {tid }} \simeq 20 \mathrm{kpc}\right.$, and $\left.r_{\text {peri }} \lesssim 10 \mathrm{kpc}\right)$. We identified infalling satellites of this 
kind in all four of the host halo histories studied (Figure 1), and such accretion events should have been ubiquitous in the history of Galaxy-sized halos since $z \sim 1$ (e.g., Stewart et al. 2008). All of these facts suggest that our simulation set has reasonably captured the global dynamical effects of halo substructure on thin galactic disks. For detailed comparisons with specific disk galaxies it would be required to explore a range of accretion histories and orbital distributions of infalling objects using a larger sample of halos from cosmological $N$-body simulations.

The analysis presented in Figures 4 and 5 highlight two distinctive signatures that infalling satellites imprint in the structure and kinematics of the host galactic disk. Figure 4 demonstrates that the thickness of the simulated disk increases with galactocentric radius, while Figure 5 illustrates that the disk velocity dispersion profiles become nearly flat at large radii ( $\left.\gtrsim 3 R_{d}\right)$. Both features appear to be a natural result of encounters with CDM substructure and have important observational consequences. The absence of these signatures in a significant fraction of disk galaxies would be difficult to reconcile in the context of the present study and could be used to potentially falsify our proposed model.

Interestingly, disk-flaring is seen in the MW in both the stellar disk (López-Corredoira et al. 2002; Momany et al. 2006) and atomic hydrogen distribution (e.g., Merrifield 1992; Nakanishi $\&$ Sofue 2003). Flaring is also observed in edge-on, external galaxies, in their stellar light (e.g., de Grijs \& Peletier 1997; Narayan \& Jog 2002) and H I gas (e.g., Brinks \& Burton 1984; Olling 1996; Matthews \& Wood 2003). Flat vertical velocity dispersion profiles are also reported in the outskirts of disk galaxies. Recently, Herrmann et al. (2009) performed a kinematic study of planetary nebulae in the extreme outer disks of the nearby, nearly face-on spirals M83 and M94. In both these systems, the kinematic evidence suggests that: (1) the stellar disks flare dramatically in their outer regions, beyond $\sim 4$ disk scale lengths; and (2) at these large distances, the vertical velocity dispersions are nearly independent of radius $\left(\sigma_{z} \sim 20 \mathrm{~km} \mathrm{~s}^{-1}\right)$ rather than decreasing exponentially as expected for a constant mass-to-light ratio, constant scale-height exponential disk. These findings are in very good agreement with the theoretical predictions presented in Figures 4 and 5, suggesting that the flaring and higher than expected values of $\sigma_{z}$ in the disks of M83 and M94 could be due to bombardment by halo substructure. Exploring these constraints further would require extending the number of kinematic surveys in the outer disks of spiral galaxies as well as performing an extensive series of numerical experiments to fully sample the statistical variation in halo accretion histories predicted by $\Lambda \mathrm{CDM}$.

Fourier analysis of their surface density illustrates that the simulated stellar disks also exhibit lopsidedness spawned by the interactions with CDM substructure (Figure 7). This has important implications as lopsided stellar disks are ubiquitous (Rix \& Zaritsky 1995; Zaritsky \& Rix 1997; Rudnick \& Rix 1998; Bournaud et al. 2005; Reichard et al. 2008). Other proposed mechanisms for generating lopsidedness have included tidal interactions and mergers (Walker et al. 1996; Zaritsky \& Rix 1997; Angiras et al. 2006, 2007; Mapelli et al. 2008), asymmetric accretion of intergalactic gas into the disk (Bournaud et al. 2005), offsets between the disk and the dark matter halo (Levine \& Sparke 1998), dynamical instabilities/processes internal to the disk (Sellwood \& Merritt 1994; Syer \& Tremaine 1996; Sellwood \& Valluri 1997; De Rijcke \& Debattista 2004; Saha et al. 2007; Dury et al. 2008), and halo lopsidedness (Jog 1997, 1999).
It is plausible that a number of mechanisms operate in concert to foster lopsidedness.

Further comparison of our results with observational work on stellar lopsidedness is worthwhile. Zaritsky \& Rix (1997) used near-infrared photometry to study a sample of 60 late-type spiral galaxies in the field. These authors defined a quantitative measure of lopsidedness as the radially averaged ratio of the $m=1$ to $m=0$ Fourier amplitudes between 1.5 and 2.5 disk scale lengths $R_{d}$, and denoted this quantity by $\left\langle A_{1}\right\rangle$. Averaging over a range of radii reduces the effect of isolated asymmetric peaks on the results of the observational analysis and provides a global measure of the lopsidedness in each galaxy. Zaritsky \& Rix (1997) found that $\sim 30 \%$ of all disk galaxies in their sample exhibit significant stellar lopsidedness with $\left\langle A_{1}\right\rangle \geqslant 0.2$. Rudnick \& Rix (1998) followed up on this work by using $R$-band photometry to investigate asymmetries in 54 early-type spirals. They reported a median value of $\left\langle A_{1}\right\rangle$ in their sample of 0.11 and that $20 \%$ of their disk galaxies had $\left\langle A_{1}\right\rangle \geqslant 0.19$. We note in passing that the similar amplitude of lopsidedness in disks with very different star formation rates indicates that the majority of the observable asymmetries in the stellar light of galactic disks reflects asymmetries in the stellar mass distribution rather than asymmetric star formation, confirming a dynamical origin of stellar lopsidedness. More recently, Bournaud et al. (2005) analyzed a sample of 149 galaxies from the Ohio State University Bright Galaxy Survey observed in the near-infrared and reported a mean $\left\langle A_{1}\right\rangle$ equal to 0.11 .

We have followed these authors and computed the quantity $\left\langle A_{1}\right\rangle$ in our simulated stellar disks. All measurements were performed after allowing the disks to relax from the encounter with each infalling subhalo as described in Section 2.4. As shown in Figure 6, the slope of the surface density profile and hence the disk scale length does not evolve significantly in the relevant radial range for measuring $\left\langle A_{1}\right\rangle, 1.5 R_{d} \lesssim R \lesssim 2.5 R_{d}$. Therefore, we adopted the scale length of the initial disk for these calculations and computed the quantity $\left\langle A_{1}\right\rangle$ in each case by averaging $A_{1}$ between $\sim 4.2 \mathrm{kpc}$ and $\sim 7 \mathrm{kpc}$. We also confirmed that using different bin numbers in calculating $\left\langle A_{1}\right\rangle$ gives similar results.

For the simulated accretion history of host halo $\mathrm{G}_{1}$, we derived $\left\langle A_{1}\right\rangle$ values in the range $0.10 \lesssim\left\langle A_{1}\right\rangle \lesssim 0.16$. For reference, the initial axisymmetric disk had $\left\langle A_{1}\right\rangle \simeq 0.003$. We stress that much larger values of $\left\langle A_{1}\right\rangle$ are excited during the subhalo impacts where tidal forces are strongest, but these are shorter-lived. As expected, the amplitude of $\left\langle A_{1}\right\rangle$ decreases steadily after the last satellite passage, reaching a value of $\sim 0.013$ in the final disk. We also found that the magnitude of the induced lopsided asymmetries depends sensitively on the structural properties of the infalling subhalos as well as those of the galactic disks. For example, satellites modeled with steep density profiles generated a significantly stronger lopsidedness compared to that of the cored counterparts. In addition, bulgeless disks develop more pronounced lopsided asymmetries in their inner regions compared to disks with massive bulges. These trends can be readily understood as a consequence of the strength of subhalo tidal perturbations onto galactic disks. Extending the mass spectrum of infalling subhalos to include the most massive systems with $M_{\text {sub }} \gtrsim M_{\text {disk }}$ (e.g., Purcell et al. 2009) would be required to examine whether satellite bombardment can explain the largest lopsided asymmetries observed in some galaxies. Additional mechanisms for lopsidedness, as discussed above, may also need to be invoked in this respect. 
The above analysis demonstrates that encounters with CDM substructure can excite lopsidedness in stellar disks at levels similar to those measured in observational samples of disk galaxies (Rix \& Zaritsky 1995; Zaritsky \& Rix 1997; Rudnick $\&$ Rix 1998). It also indicates that $\Lambda$ CDM-motivated subhalo accretion histories can maintain these lopsided asymmetries for a significant fraction of the cosmic time ( $\gtrsim 5$ Gyr). This becomes particularly relevant in light of the fact that several studies find no observed correlation between the presence of nearby companions and disk lopsidedness (e.g., Zaritsky \& Rix 1997; Wilcots \& Prescott 2004; Bournaud et al. 2005).

Our simulations also suggest that significant disk tilting may result in response to encounters with CDM subhalos (Figure 8). Disk tilting has potentially important implications. The formation of disk galaxies remains poorly understood despite some recent advances (e.g., Weil et al. 1998; Abadi et al. 2003; Sommer-Larsen et al. 2003; Governato et al. 2004, 2007; Robertson et al. 2004). The relation between the angular momenta of galactic disks and the net angular momenta of their host halos remains unclear. Dark matter halos typically have their net angular momenta aligned with the shortest of their principle axes (though the degree depends on halo mass, e.g., Bailin \& Steinmetz 2005). In the Local Group, the disks of the MW and M31 seem to have angular momenta that point along the larger-scale structure delineated by the Local Group dwarf galaxies (Majewski 1994; Hartwick 2000; Willman et al. 2004; Zentner et al. 2005b; Libeskind et al. 2005). Several studies have attempted to test for such alignments between disk principle axes and large-scale structure (Zaritsky et al. 1997; Yang et al. 2006; Azzaro et al. 2006, 2007; Bailin et al. 2008), but little evidence for either alignment have been found in statistically large samples. Our results indicate that in addition to intrinsic scatter among the angular momenta of halos and their largescale environments (Bailin \& Steinmetz 2005) such alignment may be diluted because disks, once formed, may tilt in response to numerous interactions with infalling satellites.

As far as the robustness of galactic disks to encounters with satellites is concerned, Figures 4, 5, and 9 illustrate that thicker disks are relatively more resilient to subhalo bombardment compared to their thin counterparts (see, however, Sellwood et al. 1998). Infalling satellites deposit energy into galactic disks via impulsively shocking individual orbits of disk stars during their passage ("direct heating") as well as by exciting global collective modes in the disk. Collective modes include both vertical bending waves (e.g., warps) and horizontal density waves (e.g., spiral structure and bars) and we stress that there is coupling between planar and vertical modes in three dimensions. In this case, the energy is transferred to the disk causing its heating by damping of the waves via resonant coupling (e.g., Weinberg 1991). Alternatively, the waves may be damped by dynamical friction exerted by the dark matter halo, thus yielding no heating of the disk.

Calculations of direct heating show that during a single, random orientation passage of a satellite with mass $M_{\text {sat }}$ moving at relative speed $v$ and impact parameter $b$, the mean change in the vertical energy per unit mass of a disk star is given by $\Delta E_{z}=\left(h_{z}^{2} / 3\right)\left(G M_{\text {sat }} / b^{3} \kappa_{z}\right)^{2} \beta^{2} L(\beta)$ (Spitzer 1958). Here, $h_{z}$ is the rms thickness of the disk, $\kappa_{z}$ is the frequency of vertical oscillations, the parameter $\beta=2 \kappa_{z} b / v$ is of order the characteristic passage time of the satellite divided by the orbital period of the star, and $L(\beta)$ is a dimensionless function which is unity for $\beta \rightarrow 0$ and exponentially small for $\beta \gg 1$. According to this formula, direct heating should vanish as the disk becomes razor thin, both because $h_{z} \rightarrow 0$ and also because $\kappa_{z} \rightarrow \infty$. This fact in conjunction with the results in Figures 4, 5, and 9 suggests that the relative fragility of thinner disks to encounters with satellites lies in both the ability of these systems to support global instabilities and the effectiveness of collective modes to transfer energy in this case. The absence of a bar and the much weaker warp and spiral structure induced by the subhalo impacts in galaxy model D2 compared to its thinner counterpart D1 lends support to this conjecture. Definitive investigations of these issues would require a combination of targeted numerical experiments as well as extensions of earlier analytic work (e.g., Weinberg 1989; Weinberg \& Blitz 2006).

A relevant issue concerns the existence of bulgeless, thin-disk galaxies in cosmological models where accretions of massive satellites are as common as predicted in $\Lambda \mathrm{CDM}$. Figure 10 demonstrates that a bulge component reduces significantly the damage done to the disk, so that bulgeless disk galaxies experience substantially more thickening by infalling satellites compared to their counterparts with bulges. Because most MW-sized halos are expected to have accreted numerous substructures that are a significant fraction of the disk mass including at least one system as massive as the disk since $z=1$ (Figure 1; Stewart et al. 2008), the ubiquity of very thin, bulgeless disk galaxies containing dominant old stellar populations would be difficult to reconcile with $\Lambda$ CDM. Interestingly, using SDSS data Kautsch et al. (2006) recently compiled a uniform catalog of 3169 edgeon disk galaxies and found that $\sim 1 / 3$ of the galaxies in their sample were bulgeless, "super-thin" disks with extreme axial ratios. Moreover, all systems in the sample of bulgeless, edgeon spirals of Dalcanton \& Bernstein (2002) have pronounced thick disks and there are no signs of companions in the vicinity of the prototype thin bulgeless disk galaxy M33. Formulating a comprehensive model for the formation and survivability of very thin, bulgeless disk galaxies in the context of hierarchical CDM remains challenging.

The findings of the present study as well as those of Paper I have interesting implications for the formation of thick disks. Thick disks are structurally, chemically, and kinematically distinct from thin disks, and there is evidence that they may assemble quite early in the history of a galaxy (Elmegreen \& Elmegreen 2006). Thick-disk stars in the MW and external galaxies are characterized by much larger scale heights, exhibit larger velocity dispersions and slower rotation, and are more metal-poor and significantly enhanced in $\alpha$-elements compared to thin-disk stars (e.g., Reid \& Majewski 1993; Gilmore et al. 1995; Wyse \& Gilmore 1995; Prochaska et al. 2000; Chiba \& Beers 2000; Bensby et al. 2005; Seth et al. 2005; Yoachim \& Dalcanton 2006; Allende Prieto et al. 2006; Jurić et al. 2008; Ivezić et al. 2008). While our dissipationless simulations can neither verify nor disprove any of the trends regarding metallicities, the present study does show that encounters with infalling subhalos increase considerably the scale heights and velocity ellipsoids of thin, galactic disks (Figures 4 and 5). Furthermore, the vertical structure of the final disk is well described by a standard "thin-thick" disk decomposition (see Figure 4 in Paper I) and analysis of the mean azimuthal velocity of disk stars at the solar radius in our simulations also reveals a vertical gradient in rotational velocity of $\sim-20 \mathrm{~km} \mathrm{~s}^{-1} \mathrm{kpc}^{-1}$ between 1 and $3 \mathrm{kpc}$ from the disk plane, which is consistent with what is inferred for the thick disk of the MW (Allende Prieto et al. 2006). These results suggest that at least part of a galaxy's thick-disk component may plausibly originate from the vertical dynamical heating of preexisting thin disks by CDM 
substructure. While this conclusion is supported by a number of observational studies in both the MW (e.g., Robin et al. 1996; Chen et al. 2001; Bensby et al. 2005) and external galaxies (Seth et al. 2005), more detailed theoretical modeling of the properties of thick disks would at least require the inclusion of gasdynamics, star formation, and metal enrichment.

Of course, vertical dynamical heating of an existing thin disk does not constitute the only viable model for the origin of thick disks. Other proposed mechanisms include satellite accretion events that directly deposit thick-disk stars at large scale heights (e.g., Statler 1988; Abadi et al. 2003; Yoachim \& Dalcanton 2005, 2008) as well as thick-disk stars forming in situ at early times directly from gas at large distances above the midplane (e.g., Brook et al. 2004, 2005). While there is no definitive observational or theoretical evidence to rule out any of the models for the origin of thick disks conclusively, the existence of very slowly rotating or counter-rotating thick disks in a significant fraction of disk galaxies would be particularly problematic for the "vertical heating" mechanism (Yoachim \& Dalcanton 2005). Most likely, all mechanisms do operate simultaneously at a different degree in forming the thick-disk components of galaxies.

In this paper, we have focused on the gravitational interaction between galactic disks and CDM substructure in the collisionless regime. Given the complex interplay of effects relevant to the formation and evolution of galactic disks, the inclusion of gasdynamics, star formation, and chemical evolution would be required at the least to refine the conclusions presented here. Specifically, the modeling of hydrodynamics will be crucial in determining the extent to which the presence of gas can influence the dynamical response of galactic disks to satellite accretion events and affect the properties of the final disk. For example, both subhalos and the disk may contain gas, particularly at high redshift. The satellite accretion events would then trigger bursts of star formation that may replenish a thin-disk component.

Moreover, a dissipative component may alter the dynamical effects of substructure on stellar disks in two important ways. First, the gas itself can absorb part of the orbital energy deposited into the galactic disk by the infalling systems, acting as an energy sink. This process would lead to the heating of the gaseous component. However, the efficiency of this mechanism in reducing the dynamical damage done to a stellar disk will depend on the gas content of galactic disks at early times when most of these accretion events occur. Interestingly, analytical models for the evolution of the MW disk in a cosmological context do estimate that the gaseous disk at $z \sim 1$ should amount to $\sim 50 \%$ of the mass of the stellar disk (Naab \& Ostriker 2006). Given that substantial gas fractions are expected at high redshifts, the role of gas in stabilizing the galactic disks against the violent gravitational encounters with satellites may be crucial (Stewart et al. 2009).

Second, owing to its dissipative nature, the gas can radiate its energy away. As a result, the gas that has been heated by an encounter with a subhalo can subsequently cool and reform a thin disk. As any gaseous component slowly accumulates in the center of the mass distribution, it will also induce concomitant contraction of the thickened stellar disk due to its gravity. Larger scale smooth gas accretion in galaxy formation models (e.g., Murali et al. 2002) will also be relevant in this context. A full exploration of these contingencies is challenging and we defer such studies to future work. In what follows, we present a simple, preliminary experiment to serve as a crude estimate of such effects.
In particular, we investigated the response of an initial thick disk to the adiabatic growth of a massive, thin-disk component within it. For the former, we adopted galaxy model D2 with a vertical scale height of $z_{d}=1 \mathrm{kpc}$. We considered three growing, exponential thin disks with masses $M_{\text {grow }}=$ $[0.1,0.5,1] M_{\text {disk }}$, where $M_{\text {disk }}$ is the mass of the disk in model $\mathrm{D} 2$, and a sech ${ }^{2}$ scale height of $z_{\text {thin }}=0.2 \mathrm{kpc}$. The latter value is consistent with the scale heights of known, young, star-forming disks observed in both external galaxies (e.g., Wainscoat et al. 1989; Matthews 2000) and in the MW (e.g., Bahcall \& Soneira 1980; Reid \& Majewski 1993). All growing disks were treated as rigid potentials and had the same radial scale lengths as disk D2. Furthermore, the scale length of each thin disk was kept constant while its mass was slowly increased from zero to its final value linearly over a timescale of 1 Gyr. Such timescales are in general accordance with disk formation models (e.g., Fall \& Efstathiou 1980) and ensure that the process of disk growth is approximately adiabatic.

Figure 13 presents the results of these experiments. In all cases, the initial thick disk contracts vertically as well as radially in response to the growth of the thin-disk component. Moreover, its vertical velocity dispersion increases, reflecting the deepening of the potential well due to the slow accumulation of thin-disk material. As expected, these changes in the structure of the thick disk depend sensitively on the total mass of the growing disk. In the most dramatic case with $M_{\text {grow }}=M_{\text {disk }}$, the decrease in the thickness of disk model D2 is $\sim 30 \%$ in the solar neighborhood. Furthermore, these changes do not occur uniformly as a function of radius. The disk potentials are centrally concentrated, so the evolution of the inner disk is much more pronounced compared to that of the outer disk. Overall, the results presented in Figure 13 suggest that the mass of the growing disk would need to be many times that of the thickened stellar disk to reduce its thickness appreciably, and even then, there would be stability issues.

It is important to emphasize that the aforementioned experiments were not designed to elucidate the importance of adiabatic thick-disk contraction in the specific case of the MW whose midplane density ratio of thick-to-thin disk is only $12 \%$ (e.g., Jurić et al. 2008). In a study of thick disks in the Hubble Space Telescope Ultra Deep Field (UDF), Elmegreen \& Elmegreen (2006) did examine whether such a process could have determined the present-day scale height of the thick disk of the MW. Their calculations showed that if the present thick-disk component of the MW began as an equilibrium pure-thick disk at a young age, and if subsequent accretion of the entire thin disk was adiabatic, then the initial thick-disk scale height had to be $\sim 3 \mathrm{kpc}$. This is considerably larger than that observed for young thick disks in the UDF, where the average scale height is $1.0 \pm 0.4 \mathrm{kpc}$.

\section{COMPARISON TO PREVIOUS WORK}

The response of disks to encounter with infalling satellites has received a great deal of attention owing to the numerous implications that it entails for theories of galaxy formation and evolution. Here, we discuss the main differences between our results and those reported in a subset of previous studies.

Font et al. (2001) and Gauthier et al. (2006) carried out numerical simulations of the gravitational interaction between galactic disks and a large ensemble of dark matter subhalos. Both of these investigations considered the $z=0$ satellite populations present in a MW-sized CDM halo, and both studies reached the conclusion that halo substructure has an insignificant effect on the global structure of a galactic disk. In particular, Font et al. 

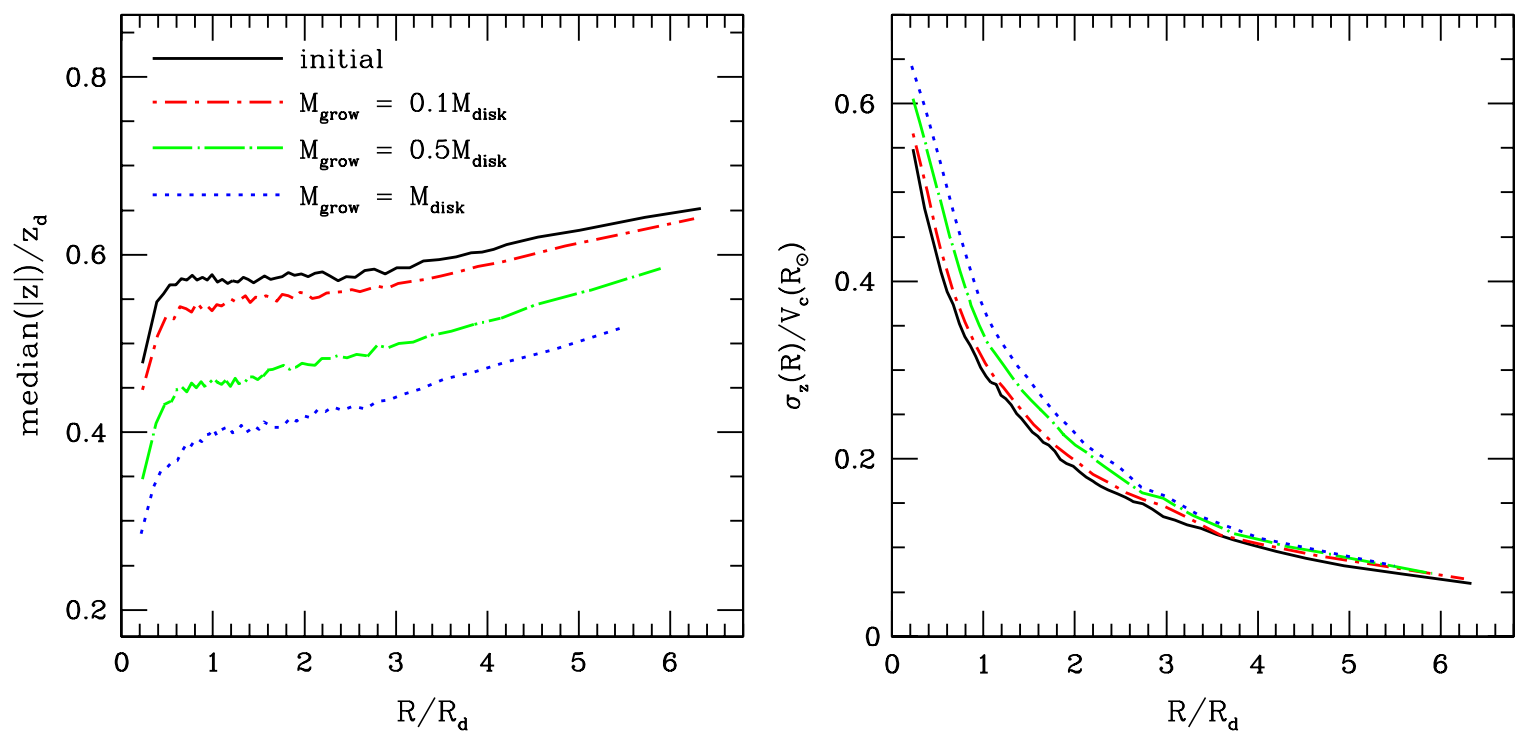

Figure 13. Effect of the adiabatic growth of a thin-disk component on the vertical structure of a thick disk. For the latter, we adopt disk model D2. The solid lines show results for the initial uncontracted thick disk with mass $M_{\text {disk }}$ while the remaining lines correspond to the final state of the thick disk after the growth of thin disks with various masses, $M_{\text {grow }}$. All growing thin disks have a sech ${ }^{2}$ scale height of $z_{\text {thin }}=0.2 \mathrm{kpc}$ and the same radial scale length, $R_{d}$, as that of the initial thick disk. Left: disk thickness profiles. Thicknesses and radii are normalized to the scale height, $z_{d}$, and radial scale length, $R_{d}$, of the initial thick disk. Right: vertical velocity dispersion profiles normalized to the total circular velocity of disk model D2 at the solar radius, $V_{\mathrm{c}}\left(R_{\odot}\right)=235.6 \mathrm{~km} \mathrm{~s}^{-1}$. The slow accumulation of thin-disk material modifies the structure of the initial thick disk, causing its vertical and radial contraction as well as an increase of its vertical velocity dispersion.

(A color version of this figure is available in the online journal.)

(2001) showed similar tidal heating rates in their $N$-body stellar disk with and without substructure, while Gauthier et al. (2006) reported appreciable heating in the inner disk regions due to the formation of a bar and only mild heating of the disk at intermediate and large radii. These results are explained by the fact that the strategies of Font et al. (2001) and Gauthier et al. (2006) excepted those systems that are most capable of strongly perturbing the disk. Massive subhalos on highly eccentric orbits at early epochs suffer substantial mass loss or become preferentially disrupted during their orbital evolution in the host potential prior to $z=0$ (Zentner \& Bullock 2003; Gao et al. 2004; Zentner et al. 2005a; Benson 2005), and so they are more likely to be absent from the present-day subhalo populations. Indeed, in the Font et al. (2001) experiments only subhalos with masses below $10^{9} M_{\odot}$ had pericenters within the solar radius, $R_{\odot}$. A primary improvement in our modeling is that we have followed the formation history of the host halo since $z \sim 1$, and consequently we have accounted for a larger number of important satellite-disk interactions than that based on the $z=0$ substructure. As a result, we report significantly more damage to the structure of the galactic disk than that demonstrated by either Font et al. (2001) or Gauthier et al. (2006).

Past numerical investigations into the resilience of galactic disks to infalling satellites have also suffered from numerical limitations and/or from assumptions which curtail their ability to accurately capture the degree of global dynamical evolution that accreting subhalos can induce in cold, stellar disks in a cosmological context. For example, the modeling of various components in the primary disk galaxy and/or the satellites as rigid potentials (e.g., Quinn \& Goodman 1986; Quinn et al. 1993; Sellwood et al. 1998; Ardi et al. 2003; Hayashi \& Chiba 2006), the initialization of a disk much thicker compared to typical thin disks such as the old, thin stellar disk of the MW (e.g., Quinn \& Goodman 1986; Quinn et al. 1993; Walker et al. 1996; Huang \& Carlberg 1997; Velazquez \& White 1999; Font et al. 2001; Villalobos \& Helmi 2008), and the infall of satellites with orbital parameters and/or structural properties inconsistent with $\Lambda$ CDM expectations (e.g., Quinn et al. 1993; Walker et al. 1996; Huang \& Carlberg 1997; Velazquez \& White 1999). For example, Quinn \& Goodman (1986), Quinn et al. (1993), and Walker et al. (1996) performed the first numerical explorations of the interaction of single satellites with larger disk galaxies. These studies unanimously found disks to be quite fragile, reporting that an encounter with a satellite of only $10 \%$ of the disk mass could increase the disk thickness by a factor of $\sim 2$ at the solar radius (Quinn \& Goodman 1986; Quinn et al. 1993). In contrast, we find galactic disks to be generally more robust to accretion events than these earlier investigations have indicated.

These differences may be due to a variety of factors. When a satellite is modeled as a distribution of interacting particles as opposed to a rigid potential, the efficiency with which it can heat a galactic disk is suppressed for two reasons. First, a selfgravitating satellite suffers mass loss due to tidal stripping and shocking. Second, a responsive satellite can absorb part of its orbital energy, decreasing the amount of energy deposited into random motions of disk stars. Moreover, live halos are needed to treat the effect of an accreting satellite on disk structure properly. Representing DM halos as rigid potentials leads to an overestimate of disk thickening by a factor of $\sim 1.5-2$, whereas a self-gravitating halo will respond to both the disk and satellite and aid in stabilizing the disk (Nelson \& Tremaine 1995; Velazquez \& White 1999). Moreover, the focus on prograde circular or nearly circular orbits in some of the aforementioned studies is also likely to have overestimated the typical amount of disk damage. The orbit with the most damaging effect for a thin disk is a coplanar, prograde circular orbit, since this causes the satellite force to be in near resonance with the disk stars. More eccentric orbits are likely to cause less damage to a disk. This is immediately apparent from the fact that, in the impulsive limit, the energy transfer scales as $\propto v^{-2}$. To a first order, the high- 
speed pericentric passages of CDM subhalos on highly eccentric orbits are thus expected to cause less thickening than circular ones. However, it is unclear to what extent highly eccentric orbits can excite global modes in the disk. This requires a detailed series of numerical experiments to evaluate.

Velazquez \& White (1999) conducted a large number of $\mathrm{N}$ body experiments in which they investigated the dependence of disk heating on, among other variables, the inclination and eccentricity of the initial satellite orbit. These authors showed that prograde encounters are more efficient at thickening the disk than retrograde ones, arguing for a resonant coupling between the satellite and disk angular momenta that is suppressed in retrograde interactions. Velazquez \& White (1999) found that a satellite with an initial mass of $M_{\text {sub }}=0.2 M_{\text {disk }}$ on a prograde orbit causes an additional increase in the scale height of the initial disk at the solar radius of $\sim 35 \%$ compared to its retrograde counterpart.

While our simulations indicate qualitatively similar differences between prograde and retrograde interactions (Figure 12), they show a less significant differential effect compared to Velazquez \& White (1999). This discrepancy has two plausible sources. First, we study cosmologically motivated, comparably high-speed passages of substructures. In such rapid ("impulsive") interactions, the resonant coupling between the satellite and disk angular momenta is expected to be weaker, explaining why the relative effect of prograde versus retrograde interactions is smaller in our experiments. Second, we study single subhalo passages, whereas Velazquez \& White (1999) followed the infalling satellites over many orbits until they merge with the galactic disk or become disrupted in the process. This is relevant because Velazquez \& White (1999) found that during the early stages of the interaction when the satellite orbit is still eccentric the difference between the amount of disk thickening caused by prograde and retrograde encounters was fairly small. Satellites on prograde orbits constituted much more efficient perturbers compared to their retrograde counterparts only during the late stages of the interaction when, owing to dynamical friction, the satellite speed has been reduced, and its orbit has both circularized and become coplanar with the disk (H. Velazquez 2008, private communication).

Toth \& Ostriker (1992), Benson et al. (2004), and Hopkins et al. (2008) quantified the fragility of galactic disks to infalling satellites by using semianalytic models of different degrees of sophistication. Analytical approaches have the advantage of not being limited by numerical resolution, allowing the calculation of a statistically large number of model realizations, but also have the drawback that they cannot account fully for the nonlinear interaction between satellites and disks. Yet, as both our results in Figure 11 suggest and other more targeted studies have indicated (e.g., Weinberg 1991; Sellwood et al. 1998), the excitation of global collective modes in the disk is an essential mechanism for energy deposition by an accreting satellite.

Toth \& Ostriker (1992) concluded that sinking satellites with only a few percent of the disk mass could lead to substantial disk thickening. Specific comparisons with the work of Toth \& Ostriker (1992) are very difficult. In a sense, the fact that their disk suffers substantial damage strengthens our findings. However,the fact that their results are averaged all possible orbital inclinations makes specific model comparisons cumbersome. Another difficulty lies in their assumption that the orbital energy of the satellite is deposited locally at the point of impact. Our results do indicate that the energy imparted by typical cosmological substructures will be deposited globally across the entire disk. Lastly, Toth \& Ostriker (1992) assumed that the total thickening and heating scale with satellite mass and that they are the same regardless of the initial thickness and velocity ellipsoid. Figures 4 and 9 show that this conjecture was also incorrect. The implications of most of these assumptions are unclear.

In contrast, Benson et al. (2004) suggested that the observed thickness of stellar disks is entirely compatible with the abundance of substructure in CDM halos. In addition to not accounting for global collective modes, Benson et al. (2004) also adopted satellite orbits that spanned only a limited range of orbital energies and angular momenta in spherical halo potentials rather than the richer variety of impacts experienced over the course of halo formation (e.g., Zentner et al. 2005a, 2005b; Benson 2005). Consequently, this study suffers from a flaw similar to that of Font et al. (2001) and Gauthier et al. (2006), explaining possibly why we report more significant damage to the disk structure by subhalo bombardment.

More direct comparison can be performed with the recent work of Hopkins et al. (2008). These authors have argued that deposition of orbital energy into the disk in the context of realistically radial subhalo orbits and $\Lambda$ CDM-motivated accretion histories would yield a much less dramatic impact on the disk structure than previously thought, with or without the presence of gas. In particular, Hopkins et al. (2008) derived that the disk heating efficiency is nonlinear in mass ratio, $\propto\left(M_{\text {sub }} / M_{\text {disk }}\right)^{2}$, instead of the linear scaling of Toth \& Ostriker (1992), implying that the fractional change in disk scale height should be very small even for the very massive subhalos we considered in the present study $\left(0.2 M_{\text {disk }} \lesssim M_{\text {sub }} \lesssim M_{\text {disk }}\right)$. Hopkins et al. (2008) defined a disk thickening parameter $\Delta H / R$, where $H$ is the median disk scale height and $R$ is the radius where the scale height is measured, typically within a factor of 2 of the disk half-mass radius, $R_{h}$.

For the simulated accretion history of host halo $G_{1}$, the Hopkins et al. (2008) formula predicts $\Delta H / R \simeq 0.01$, if we simply add the masses of all cosmological subhalos S1-S6. Our numerical experiments indicate significantly more thickening. Indeed, at $R=R_{h}=1.7 R d$, we measure $\Delta H / R \simeq 0.02$, and because of the pronounced flaring in response to the accretion events, we report even larger $\Delta H / R \simeq 0.04$, at $R=2 R_{h}$. A variety of reasons may be responsible for these discrepancies. As before, global collective modes which may dominate the disk response to accretion events were not included in these simple analytic scalings. Furthermore, Hopkins et al. (2008) calibrated their results to numerical simulations (Velazquez \& White 1999; Villalobos \& Helmi 2008) with initial disks that were significantly thicker compared to the thin, galactic disk we employed here, and were therefore intrinsically more robust to encounters with satellites (Figure 11).

Lastly, special emphasis should be placed on the recent studies by Read et al. (2008) and Purcell et al. (2009). These authors performed collisionless $N$-body simulations to study the response of an initially thin $\left(z_{d} \simeq 400 \mathrm{pc}\right)$, MW type disk galaxy to $\sim 1: 10$ satellite impacts. Such accretion events represent the primary concern for disk survival in the $\Lambda \mathrm{CDM}$ cosmological model and should have been commonplace in the history of Galaxy-sized halos (Stewart et al. 2008). Purcell et al. (2009) quantitatively demonstrated the destruction of a thin, stellar disk by these cosmologically motivated common events. They found that regardless of orbital configuration, the impacts transform the disks into structures that are roughly three times as thick and more than twice as kinematically hot as the observed 
dominant old thin-disk component of the MW. On the other hand, our work shows that a thin-disk component may survive, even strongly perturbed, the encounters with halo substructure (Paper I). This is because we have focused on infalling systems with masses in the range $0.2 M_{\text {disk }} \lesssim M_{\text {sub }} \lesssim M_{\text {disk }}$, ignoring the most massive accretion events that could prove ruinous to thin-disk survival. Our findings are thus relevant to systems that have already experienced the most destructive events simulated by Purcell et al. (2009) since $z=1$ and have re-grown their thin disks since. In this sense, our simulation set and results are complementary to those of Purcell et al. (2009).

\section{SUMMARY}

Using a suite of high-resolution, fully self-consistent dissipationless $N$-body simulations we have examined the dynamical effects of halo substructure on thin galactic disks in the context of the $\Lambda \mathrm{CDM}$ paradigm of structure formation. Our simulation campaign utilizes cosmological simulations of the formation of Galaxy-sized CDM halos to derive the properties of infalling subhalo populations and controlled numerical experiments of consecutive satellite encounters with an initially thin, fully formed disk galaxy. As a corollary, we have quantified the importance of various physical effects that could influence the response of a galactic disk to substructure accretion events. The properties we address are the initial disk thickness, the presence of a bulge component in the primary galaxy, the internal density distribution of the infalling systems, and the relative orientation of disk and satellite angular momenta.

Our work expands upon past numerical investigations into the dynamical response of galactic disks to accreting satellites in several ways. One improvement concerns the more realistic treatment of the infalling subhalo populations. Previous studies of the interaction between disks and ensembles of subhalos considered only the $z=0$ surviving substructure present in a CDM halo (Font et al. 2001; Gauthier et al. 2006). This leads to estimates of the damage done to the disk that are biased low, because massive subhalos with small orbital pericenters that are most capable of strongly perturbing the disk are preferential removed from the satellite populations over time. We have accounted for satellite-disk interactions with infalling subhalos that typically do not survive to the present day but nevertheless cause significant damage to the disk. This is the major conceptual advantage of our work and this difference drives our initial disks to be more dramatically affected by halo substructure than those in earlier studies. Second, the primary disk galaxy models we utilize are fully self-consistent particle realizations derived from explicit DFs and are designed to satisfy a broad range of observational constraints available for actual disk galaxies. Finally, we represent satellite systems by equilibrium numerical realizations, whose properties (mass functions, orbital parameters, internal structures, and accretion times) are extracted directly from cosmological simulations of the formation of Galaxy-sized CDM halos.

Our main conclusions can be summarized as follows.

1. Close encounters between massive satellites and galactic disks since $z=1$ are common occurrences in the $\Lambda$ CDM cosmological model. Statistics of four Galaxy-sized CDM halos indicate that, on average, $\sim 5$ subhalos more massive than $0.2 M_{\text {disk }}$, where $M_{\text {disk }}$ is the present-day mass of the stellar disk of the MW, pass within $\sim 20 \mathrm{kpc}$ from the centers of their host halos in the past $\sim 8 \mathrm{Gyr}$. In contrast, very few satellites in present-day substructure populations are likely to have a significant impact on the structure of a galactic disk. This is because massive subhalos on potentially damaging orbits suffer severe mass loss or become tidally disrupted prior to $z=0$ as a result of penetrating deeply into the central regions of their hosts (Section 2.2).

2. A conservative subset of one host halo accretion history was used to seed controlled subhalo-disk encounter simulations. The specific merger history involved the accretion of six satellites with masses, orbital pericenters, and tidal radii of $7.4 \times 10^{9} \lesssim M_{\text {sub }} / M_{\odot} \lesssim 2 \times 10^{10}, r_{\text {peri }} \lesssim 20 \mathrm{kpc}$, and $r_{\text {tid }} \gtrsim 20 \mathrm{kpc}$, respectively, since $z=1$. These events severely perturb an initially thin $\left(z_{d}=0.4 \mathrm{kpc}\right)$, MW type disk galaxy $\left(M_{\text {disk }} \approx 3.5 \times 10^{10} M_{\odot}\right)$ and imprint the following distinctive dynamical signatures on its structure and kinematics.

a) The development of nonaxisymmetric structures including a warp, a moderately strong bar, and extended ring-like features in the outskirts of the disk (Section 3.1).

b) Considerable thickening and heating at all radii, with a factor of $\sim 2$ increase in disk thickness and velocity ellipsoid at the solar radius (Sections 3.2 and 3.3).

c) Prominent flaring associated with an increase of disk thickness greater than a factor of 4 in the disk outskirts (Section 3.2).

d) Surface density excesses at large radii, beyond $\sim 5$ disk scale lengths, as observed in antitruncated disks (Section 3.4).

e) Long-lived, lopsidedness at levels similar to those measured in observational samples of disk galaxies (Section 3.5).

f) Substantial tilting of the disk from its initial orientation in the host halo, resulting in a misalignment between halo and disk principal axes and angular momenta (Section 3.6).

3. Detailed predictions for the dynamical response of galactic disks to subhalo bombardment are subject to a variety of assumptions including the initial structures of the disk and infalling systems, the prominence of the bulge component in the primary disk, and the relative orientation of disk and satellite angular momenta (Section 4).

We close with a few words of caution and a discussion of fruitful directions for future work that may lead to more conclusive statements about the detailed structure of disk galaxies. We reiterate that we have only addressed the gravitational interaction of galactic disks and halo substructure in the collisionless regime. A full consideration to the rich structure of perturbed galactic disks is challenging and would require detailed knowledge of galaxy star formation histories, gas cooling and feedback, among other things. However, such studies that treat both the gaseous components of the disk and subhalos, and accreted stars will be fundamental in refining our understanding of disk galaxy evolution and we plan to extend the present investigation in this direction.

Specifically, spiral galaxies contain atomic and molecular gas in their disks which can absorb and subsequently radiate away some of the orbital energy deposited by the sinking satellites. Thus, including a dissipative component in the primary galaxy will allow for an estimate of the extent to which the presence of gas can reduce the damage done to disks and stabilize them against the violent gravitational encounters with satellites. 
Adding star formation as a further ingredient will provide the opportunity to analyze any reformed thin disk and establish the degree to which contraction subsequent to gas cooling can decrease the thickness of a heated disk (Figure 13). The effects of gasdynamics and star formation will offer the possibility to determine the magnitude of starbursts induced in the disk as a result of subhalo bombardment, while gaining a deeper understanding of the build-up of the inner stellar halos of galaxies (Bullock \& Johnston 2005). Lastly, the inclusion of baryonic components in the satellites will enable studies whereby, original disk stars, newly formed stars, and accreted stars can be located and studied in their final configurations.

Such predictions will be vital as instruments and surveys like SDSS III, RAVE, GAIA, SIM, Pan-STARRS, LSST, and TMT are poised to provide spatial and kinematic maps of the MW and other local volume galaxies to unprecedented detail and depth. Our simulations suggest that these experiments should uncover detailed disk structure that is substantially perturbed via interactions with infalling satellites. Our ability to interpret these data sets will rely on a comprehensive set of theoretical predictions for how galactic disks respond to accretion events and how this process is convolved with direct stellar and gaseous accretions (either via the same encounters or other delivery process). In this sense, detailed probes of the local volume offer a valuable and unique avenue for constraining the process of disk galaxy formation and galaxy formation in general.

We thank the anonymous referee for comments that improved the clarity of the manuscript. The authors are grateful to Mario Abadi, Andrew Benson, Simone Callegari, Annette Ferguson, Andreea Font, Kimberly Herrmann, Kathryn Johnston, Mario Jurić, Lucio Mayer, Ben Moore, Heather Morrison, Julio Navarro, Panos Patsis, Chris Purcell, Tom Quinn, Joachim Stadel, Octavio Valenzuela, Hector Velazquez, and David Weinberg for many stimulating discussions. S.K. thanks Frank van den Bosch for communicating unpublished results, and John Dubinski and Larry Widrow for kindly making available the software used to set up the primary galaxy models. S.K., J.S.B., and A.V.K. acknowledge the Aspen Center for Physics for hosting the summer workshop "Deconstructing the Local GroupDissecting Galaxy Formation in our Own Background" during the initial stages of this work. S.K. is also grateful to the Kavli Institute for Theoretical Physics for organizing the stimulating workshop "Building the Milky Way" and for its hospitality while this work was in progress. S.K. is supported by the Center for Cosmology and Astro-Particle Physics (CCAPP) at The Ohio State University. A.R.Z. is funded by the University of Pittsburgh and by the National Science Foundation (NSF) through grant AST 0806367. J.S.B. is supported by NSF grants AST 05-07916 and AST 06-07377. A.V.K. is supported by the NSF grants AST-0239759 and AST-0507596, and by KICP. The numerical simulations were performed on the zBox supercomputer at the University of Zürich and on the Cosmos cluster at the Jet Propulsion Laboratory (JPL). Cosmos was provided by funding from the JPL Office of the Chief Information Officer. This research made use of the NASA Astrophysics Data System.

\section{REFERENCES}

Abadi, M. G., Navarro, J. F., Steinmetz, M., \& Eke, V. R. 2003, ApJ, 591, 499 Allende Prieto, C., Beers, T. C., Wilhelm, R., Newberg, H. J., Rockosi, C. M., Yanny, B., \& Lee, Y. S. 2006, ApJ, 636, 804

Angiras, R. A., Jog, C. J., Dwarakanath, K. S., \& Verheijen, M. A. W. 2007, MNRAS, 378, 276
Angiras, R. A., Jog, C. J., Omar, A., \& Dwarakanath, K. S. 2006, MNRAS, 369 1849

Ardi, E., Tsuchiya, T., \& Burkert, A. 2003, ApJ, 596, 204

Avila Reese, V., Colín, P., Valenzuela, O., D’Onghia, E., \& Firmani, C. 2001, ApJ, 559, 516

Azzaro, M., Patiri, S. G., Prada, F., \& Zentner, A. R. 2007, MNRAS, 376, L43

Azzaro, M., Zentner, A. R., Prada, F., \& Klypin, A. A. 2006, ApJ, 645, 228

Bahcall, J. N., \& Soneira, R. M. 1980, ApJS, 44, 73

Bailin, J., Power, C., Norberg, P., Zaritsky, D., \& Gibson, B. K. 2008, MNRAS, 390, 1133

Bailin, J., \& Steinmetz, M. 2005, ApJ, 627, 647

Baldwin, J. E., Lynden Bell, D., \& Sancisi, R. 1980, MNRAS, 193, 313

Belokurov, V., et al. 2006, ApJ, 642, L137

Bensby, T., Feltzing, S., Lundström, I., \& Ilyin, I. 2005, A\&A, 433, 185

Benson, A. J. 2005, MNRAS, 358, 551

Benson, A. J., Lacey, C. G., Frenk, C. S., Baugh, C. M., \& Cole, S. 2004, MNRAS, 351, 1215

Binney, J., \& Tremaine, S. 1987, Galactic Dynamics (Princeton, NJ: Princeton Univ. Press)

Bissantz, N., \& Gerhard, O. 2002, MNRAS, 330, 591

Bizyaev, D., \& Mitronova, S. 2002, A\&A, 389, 795

Blumenthal, G. R., Faber, S. M., Flores, R., \& Primack, J. R. 1986, ApJ, 301, 27

Blumenthal, G. R., Faber, S. M., Primack, J. R., \& Rees, M. J. 1984, Nature, 311,517

Bournaud, F., Combes, F., Jog, C. J., \& Puerari, I. 2005, A\&A, 438, 507

Brinks, E., \& Burton, W. B. 1984, A\&A, 141, 195

Brook, C. B., Gibson, B. K., Martel, H., \& Kawata, D. 2005, ApJ, 630, 298

Brook, C. B., Kawata, D., Gibson, B. K., \& Freeman, K. C. 2004, ApJ, 612, 894

Bullock, J. S., \& Johnston, K. V. 2005, ApJ, 635, 931

Chen, B., et al. 2001, ApJ, 553, 184

Chiba, M., \& Beers, T. C. 2000, AJ, 119, 2843

Choi, Y.-Y., Park, C., \& Vogeley, M. S. 2007, ApJ, 658, 884

Dalcanton, J. J., \& Bernstein, R. A. 2002, AJ, 124, 1328

Debattista, V. P., Mayer, L., Carollo, C. M., Moore, B., Wadsley, J., \& Quinn, T. 2006, ApJ, 645, 209

Debattista, V. P., \& Sellwood, J. A. 2000, ApJ, 543, 704

de Grijs, R. 1998, MNRAS, 299, 595

de Grijs, R., \& Peletier, R. F. 1997, A\&A, 320, L21

Dehnen, W., \& Binney, J. 1998, MNRAS, 294, 429

De Rijcke, S., \& Debattista, V. P. 2004, ApJ, 603, L25

Dury, V., de Rijcke, S., Debattista, V. P., \& Dejonghe, H. 2008, MNRAS, 387, 2

Elmegreen, B. G., \& Elmegreen, D. M. 2006, ApJ, 650, 644

Erwin, P., Beckman, J. E., \& Pohlen, M. 2005, ApJ, 626, L81

Erwin, P., Pohlen, M., \& Beckman, J. E. 2008, AJ, 135, 20

Fall, S. M., \& Efstathiou, G. 1980, MNRAS, 193, 189

Ferguson, A. M. N., Irwin, M. J., Ibata, R. A., Lewis, G. F., \& Tanvir, N. R. 2002, AJ, 124, 1452

Ferguson, A. M. N., et al. 2005, ApJ, 622, L109

Font, A. S., Navarro, J. F., Stadel, J., \& Quinn, T. 2001, ApJ, 563, L1

Forbes, D. A., Beasley, M. A., Bekki, K., Brodie, J. P., \& Strader, J. 2003, Science, 301, 1217

Gao, L., White, S. D. M., Jenkins, A., Stoehr, F., \& Springel, V. 2004, MNRAS, 355,819

Gauthier, J.-R., Dubinski, J., \& Widrow, L. M. 2006, ApJ, 653, 1180

Ghigna, S., Moore, B., Governato, F., Lake, G., Quinn, T., \& Stadel, J. 1998, MNRAS, 300, 146

Ghigna, S., Moore, B., Governato, F., Lake, G., Quinn, T., \& Stadel, J. 2000, ApJ, 544,616

Gilmore, G., Wyse, R. F. G., \& Jones, J. B. 1995, AJ, 109, 1095

Gnedin, N. Y., \& Kravtsov, A. V. 2006, ApJ, 645, 1054

Governato, F., et al. 2004, ApJ, 607, 688

Governato, F., et al. 2007, MNRAS, 374, 1479

Grillmair, C. J., \& Dionatos, O. 2006, ApJ, 643, L17

Grosbol, P. J., \& Patsis, P. A. 1998, A\&A, 336, 840

Hartwick, F. D. A. 2000, AJ, 119, 2248

Hayashi, H., \& Chiba, M. 2006, PASJ, 58, 835

Hayashi, E., Navarro, J. F., Taylor, J. E., Stadel, J., \& Quinn, T. 2003, ApJ, 584, 541

Haynes, M. P., van Zee, L., Hogg, D. E., Roberts, M. S., \& Maddalena, R. J. 1998, AJ, 115, 62

Hernquist, L. 1990, ApJ, 356, 359

Herrmann, K. A., Ciardullo, R., \& Sigurdsson, S. 2009, ApJ, 693, L19

Hogan, C. J., \& Dalcanton, J. J. 2000, Phys. Rev. D, 62, 063511

Hopkins, P. F., Hernquist, L., Cox, T. J., Younger, J. D., \& Besla, G. 2008, ApJ, 688,757

Huang, S., \& Carlberg, R. G. 1997, ApJ, 480, 503 
Ibata, R. A., Gilmore, G., \& Irwin, M. J. 1994, Nature, 370, 194

Ibata, R., Irwin, M., Lewis, G., Ferguson, A. M. N., \& Tanvir, N. 2001a, Nature, 412, 49

Ibata, R., Lewis, G. F., Irwin, M., Totten, E., \& Quinn, T. 2001b, ApJ, 551, 294

Ibata, R., Martin, N. F., Irwin, M., Chapman, S., Ferguson, A. M. N., Lewis, G. F., \& McConnachie, A. W. 2007, ApJ, 671, 1591

Ivezić, Ž., et al. 2008, ApJ, 684, 287

Jog, C. J. 1997, ApJ, 488, 642

Jog, C. J. 1999, ApJ, 522, 661

Jurić, M., et al. 2008, ApJ, 673, 864

Kalirai, J. S., Guhathakurta, P., Gilbert, K. M., Reitzel, D. B., Majewski, S. R., Rich, R. M., \& Cooper, M. C. 2006, ApJ, 641, 268

Kautsch, S. J., Grebel, E. K., Barazza, F. D., \& Gallagher, J. S., III. 2006, A\&A, 445,765

Kazantzidis, S., Bullock, J. S., Zentner, A. R., Kravtsov, A. V., \& Moustakas, L. A. 2008, ApJ, 688, 254

Kazantzidis, S., Magorrian, J., \& Moore, B. 2004a, ApJ, 601, 37

Kazantzidis, S., Mayer, L., Mastropietro, C., Diemand, J., Stadel, J., \& Moore, B. 2004b, ApJ, 608, 663

Kazantzidis, S., Zentner, A. R., \& Nagai, D. 2006, in EAS Publications Series, ed. G. A. Mamon, F. Combes, C. Deffayet, \& B. Fort (Noordwijk: ESA), 65

Kent, S. M., Dame, T. M., \& Fazio, G. 1991, ApJ, 378, 131

Klypin, A., Gottlöber, S., Kravtsov, A. V., \& Khokhlov, A. M. 1999a, ApJ, 516, 530

Klypin, A. A., Kravtsov, A. V., Bullock, J. S., \& Primack, J. R. 2001, ApJ, 554, 903

Klypin, A., Kravtsov, A. V., Valenzuela, O., \& Prada, F. 1999b, ApJ, 522, 82

Kravtsov, A. V. 1999, PhD thesis, New Mexico State Univ.

Kravtsov, A. V., Gnedin, O. Y., \& Klypin, A. A. 2004, ApJ, 609, 482

Kravtsov, A. V., Klypin, A. A., Bullock, J. S., \& Primack, J. R. 1998, ApJ, 502, 48

Kravtsov, A. V., Klypin, A. A., \& Khokhlov, A. M. 1997, ApJS, 111, 73

Kregel, M., van der Kruit, P. C., \& de Grijs, R. 2002, MNRAS, 334, 646

Kuhlen, M., Diemand, J., \& Madau, P. 2007, ApJ, 671, 1135

Lacey, C., \& Cole, S. 1993, MNRAS, 262, 627

Larsen, J. A., \& Humphreys, R. M. 2003, AJ, 125, 1958

Levine, S. E., \& Sparke, L. S. 1998, ApJ, 496, L13

Libeskind, N. I., Cole, S., Frenk, C. S., Okamoto, T., \& Jenkins, A. 2007, MNRAS, 374, 16

Libeskind, N. I., Frenk, C. S., Cole, S., Helly, J. C., Jenkins, A., Navarro, J. F., \& Power, C. 2005, MNRAS, 363, 146

López-Corredoira, M., Cabrera-Lavers, A., Garzón, F., \& Hammersley, P. L. 2002, A\&A, 394, 883

Majewski, S. R. 1994, ApJ, 431, L17

Majewski, S. R., Skrutskie, M. F., Weinberg, M. D., \& Ostheimer, J. C. 2003, ApJ, 599, 1082

Malin, D., \& Hadley, B. 1997, PASA, 14, 52

Mapelli, M., Moore, B., \& Bland-Hawthorn, J. 2008, MNRAS, 388, 697

Martin, N. F., Ibata, R. A., Bellazzini, M., Irwin, M. J., Lewis, G. F., \& Dehnen, W. 2004, MNRAS, 348, 12

Martínez-Delgado, D., Butler, D. J., Rix, H.-W., Franco, V. I., Peñarrubia, J., Alfaro, E. J., \& Dinescu, D. I. 2005, ApJ, 633, 205

Matthews, L. D. 2000, AJ, 120, 1764

Matthews, L. D., van Driel, W., \& Gallagher, J. S., III. 1998, AJ, 116, 1169

Matthews, L. D., \& Wood, K. 2003, ApJ, 593, 721

Mayer, L., Governato, F., \& Kaufmann, T. 2008, Invited Review in "Advanced Science Letters" (arXiv:0801.3845)

Mayer, L., Kazantzidis, S., Mastropietro, C., \& Wadsley, J. 2007, Nature, 445, 738

Mendez, R. A., \& Guzman, R. 1998, A\&A, 333, 106

Merrifield, M. R. 1992, AJ, 103, 1552

Meza, A., Navarro, J. F., Abadi, M. G., \& Steinmetz, M. 2005, MNRAS, 359, 93

Momany, Y., Zaggia, S., Gilmore, G., Piotto, G., Carraro, G., Bedin, L. R., \& de Angeli, F. 2006, A\&A, 451, 515

Moore, B., Ghigna, S., Governato, F., Lake, G., Quinn, T., Stadel, J., \& Tozzi, P. 1999, ApJ, 524, L19

Moore, B., Katz, N., \& Lake, G. 1996, ApJ, 457, 455

Moore, B., Kazantzidis, S., Diemand, J., \& Stadel, J. 2004, MNRAS, 354, 522

Murali, C., Katz, N., Hernquist, L., Weinberg, D. H., \& Davé, R. 2002, ApJ, 571,1

Naab, T., \& Ostriker, J. P. 2006, MNRAS, 366, 899

Nakanishi, H., \& Sofue, Y. 2003, PASJ, 55, 191

Narayan, C. A., \& Jog, C. J. 2002, A\&A, 390, L35

Navarro, J. F., Frenk, C. S., \& White, S. D. M. 1996, ApJ, 462, 563

Nelson, R. W., \& Tremaine, S. 1995, MNRAS, 275, 897
Newberg, H. J., et al. 2002, ApJ, 569, 245

Nordström, B., et al. 2004, A\&A, 418, 989

Olling, R. P. 1996, AJ, 112, 457

Peñarrubia, J., Kroupa, P., \& Boily, C. M. 2002, MNRAS, 333, 779

Peng, E. W., Ford, H. C., Freeman, K. C., \& White, R. L. 2002, AJ, 124, 3144

Pohlen, M., Martínez-Delgado, D., Majewski, S., Palma, C., Prada, F., \& Balcells, M. 2004, in ASP Conf. Ser. 327, Satellites and Tidal Streams, ed. F. Prada, D. Martinez Delgado, \& T. J. Mahoney (San Francisco, CA: ASP), 288

Pohlen, M., \& Trujillo, I. 2006, A\&A, 454, 759

Pohlen, M., Zaroubi, S., Peletier, R. F., \& Dettmar, R.-J. 2007, MNRAS, 378, 594

Prada, F., Klypin, A. A., Simonneau, E., Betancort-Rijo, J., Patiri, S., Gottlöber, S., \& Sanchez-Conde, M. A. 2006, ApJ, 645, 1001

Prochaska, J. X., Naumov, S. O., Carney, B. W., McWilliam, A., \& Wolfe, A. M. 2000, AJ, 120, 2513

Purcell, C. W., Bullock, J. S., \& Zentner, A. R. 2007, ApJ, 666, 20

Purcell, C. W., Kazantzidis, S., \& Bullock, J. S. 2009, ApJ, 694, L98

Quillen, A. C., \& Garnett, D. R. 2000, arXiv astrophysics e-prints

Quinn, P. J., \& Goodman, J. 1986, ApJ, 309, 472

Quinn, P. J., Hernquist, L., \& Fullagar, D. P. 1993, ApJ, 403, 74

Read, J. I., Lake, G., Agertz, O., \& Debattista, V. P. 2008, MNRAS, 389, 1041

Reichard, T. A., Heckman, T. M., Rudnick, G., Brinchmann, J., \& Kauffmann, G. 2008, ApJ, 677, 186

Reid, N., \& Majewski, S. R. 1993, ApJ, 409, 635

Richter, O.-G., \& Sancisi, R. 1994, A\&A, 290, L9

Rix, H.-W., \& Zaritsky, D. 1995, ApJ, 447, 82

Robertson, B., Yoshida, N., Springel, V., \& Hernquist, L. 2004, ApJ, 606, 32

Robin, A. C., Haywood, M., Creze, M., Ojha, D. K., \& Bienayme, O. 1996, A\&A, 305, 125

Rudnick, G., \& Rix, H.-W. 1998, AJ, 116, 1163

Saha, K., Combes, F., \& Jog, C. J. 2007, MNRAS, 382, 419

Schommer, R. A., Suntzeff, N. B., Olszewski, E. W., \& Harris, H. C. 1992, AJ, 103,447

Seabroke, G. M., \& Gilmore, G. 2007, MNRAS, 380, 1348

Sellwood, J. A., \& Merritt, D. 1994, ApJ, 425, 530

Sellwood, J. A., Nelson, R. W., \& Tremaine, S. 1998, ApJ, 506, 590

Sellwood, J. A., \& Valluri, M. 1997, MNRAS, 287, 124

Seth, A. C., Dalcanton, J. J., \& de Jong, R. S. 2005, AJ, 130, 1574

Shang, E., et al. 1998, ApJ, 504, L23

Shen, J., \& Sellwood, J. A. 2006, MNRAS, 370, 2

Sommer-Larsen, J., Götz, M., \& Portinari, L. 2003, ApJ, 596, 47

Soubiran, C., Bienaymé, O., \& Siebert, A. 2003, A\&A, 398, 141

Spitzer, L. J. 1958, ApJ, 127, 17

Stadel, J. G. 2001, PhD thesis, Univ. Washington

Statler, T. S. 1988, ApJ, 331, 71

Stewart, K. R., Bullock, J. S., Wechsler, R. H., \& Maller, A. H. 2009, ApJ, submitted (arXiv:0901.4336)

Stewart, K. R., Bullock, J. S., Wechsler, R. H., Maller, A. H., \& Zentner, A. R. 2008, ApJ, 683, 597

Syer, D., \& Tremaine, S. 1996, MNRAS, 281, 925

Tormen, G., Diaferio, A., \& Syer, D. 1998, MNRAS, 299, 728

Toth, G., \& Ostriker, J. P. 1992, ApJ, 389, 5

Velazquez, H., \& White, S. D. M. 1999, MNRAS, 304, 254

Villalobos, Á., \& Helmi, A. 2008, MNRAS, 391, 1806

Wainscoat, R. J., Freeman, K. C., \& Hyland, A. R. 1989, ApJ, 337, 163

Walker, I. R., Mihos, J. C., \& Hernquist, L. 1996, ApJ, 460, 121

Wechsler, R. H., Bullock, J. S., Primack, J. R., Kravtsov, A. V., \& Dekel, A. 2002, ApJ, 568, 52

Weil, M. L., Eke, V. R., \& Efstathiou, G. 1998, MNRAS, 300, 773

Weinberg, M. D. 1989, MNRAS, 239, 549

Weinberg, M. D. 1991, ApJ, 373, 391

Weinberg, M. D., \& Blitz, L. 2006, ApJ, 641, L33

Weinmann, S. M., van den Bosch, F. C., Yang, X., \& Mo, H. J. 2006, MNRAS, 366,2

White, S. D. M., \& Rees, M. J. 1978, MNRAS, 183, 341

Widrow, L. M., \& Dubinski, J. 2005, ApJ, 631, 838

Wilcots, E. M., \& Prescott, M. K. M. 2004, AJ, 127, 1900

Willman, B., Governato, F., Dalcanton, J. J., Reed, D., \& Quinn, T. 2004, MNRAS, 353, 639

Wyse, R. F. G. 2001, in ASP Conf. Ser. 230, Galaxy Disks and Disk Galaxies, ed. J. G. Funes \& E. M. Corsini (San Francisco, CA: ASP), 71

Wyse, R. F. G., \& Gilmore, G. 1995, AJ, 110, 2771

Yang, X., van den Bosch, F. C., Mo, H. J., Mao, S., Kang, X., Weinmann, S. M., Guo, Y., \& Jing, Y. P. 2006, MNRAS, 369, 1293

Yanny, B., et al. 2000, ApJ, 540, 825 
Yoachim, P., \& Dalcanton, J. J. 2005, ApJ, 624, 70

Yoachim, P., \& Dalcanton, J. J. 2006, AJ, 131, 226

Yoachim, P., \& Dalcanton, J. J. 2008, ApJ, 682, 1004

Zaritsky, D., \& Rix, H.-W. 1997, ApJ, 477, 118

Zaritsky, D., Smith, R., Frenk, C. S., \& White, S. D. M. 1997, ApJ, 478, L53
Zentner, A. R., Berlind, A. A., Bullock, J. S., Kravtsov, A. V., \& Wechsler, R. H. 2005a, ApJ, 624, 505

Zentner, A. R., \& Bullock, J. S. 2003, ApJ, 598, 49

Zentner, A. R., Kravtsov, A. V., Gnedin, O. Y., \& Klypin, A. A. 2005b, ApJ, 629, 219

Zhao, H. 1996, MNRAS, 278, 488 